\title{
INTERACTING SUPERNOVAE AND SUPERNOVA IMPOSTORS: SN 2009ip, IS THIS THE END?
}

\author{
A. Pastorello ${ }^{1}$, E. Cappellaro ${ }^{1}$, C. Inserra ${ }^{2}$, S. J. Smarti ${ }^{2}$, G. Pignata ${ }^{3}$, S. Benetti ${ }^{1}$, S. Valenti ${ }^{4,5}$, M. Fraser ${ }^{2}$, \\ K. Takáts ${ }^{3,6}$, S. Benitez ${ }^{7}$, M. T. Botticella ${ }^{8}$, J. Brimacombe ${ }^{9}$, F. Bufano ${ }^{3}$, F. Cellier-Holzem ${ }^{10}$, M. T. Costado $^{11}$, \\ G. Cupani ${ }^{12}$, I. Curtis ${ }^{13}$, N. Elias-Rosa ${ }^{14}$, M. Ergon ${ }^{15}$, J. P. U. Fynbo ${ }^{16}$, F.-J. Hambsch ${ }^{17}$, M. HamuY ${ }^{18}$, A. Harutyunyan ${ }^{19}$, \\ K. M. Ivarson ${ }^{20}$, E. Kankare ${ }^{21}$, J. C. Martin ${ }^{22}$, R. KotaK ${ }^{2}$, A. P. LaCluyze ${ }^{20}$, K. Maguire ${ }^{23}$, S. Mattila ${ }^{24}$, J. MaZA $^{18}$, \\ M. McCrum ${ }^{2}$, M. Miluzio ${ }^{25}$, H. U. NorgaArd-Nielsen ${ }^{16}$, M. C. Nysewander ${ }^{20}$, P. OChner ${ }^{1}$, Y.-C. Pan ${ }^{23}$, M. L. Pumo ${ }^{1}$, \\ D. E. Reichart ${ }^{20}$, T. G. TAN ${ }^{26}$, S. TAubenberger ${ }^{7}$, L. Tomasella ${ }^{1}$, M. Turatto ${ }^{1}$, And D. Wright ${ }^{2}$ \\ ${ }^{1}$ INAF-Osservatorio Astronomico di Padova, Vicolo dell'Osservatorio 5, I-35122 Padova, Italy; andrea.pastorello@ oapd.inaf.it \\ ${ }^{2}$ Astrophysics Research Centre, School of Mathematics and Physics, Queen's University Belfast, Belfast BT7 1NN, UK \\ ${ }^{3}$ Departamento de Ciencias Fisicas, Universidad Andres Bello, Avda. Republica 252, Santiago, Chile \\ ${ }^{4}$ Las Cumbres Observatory Global Telescope Network Inc., Santa Barbara, CA 93117, USA \\ ${ }^{5}$ Department of Physics, University of California Santa Barbara, Santa Barbara, CA 93106-9530, USA \\ ${ }^{6}$ Department of Optics \& Quantum Electronics, University of Szeged, Dóm tér 9, Szeged H-6720, Hungary \\ ${ }^{7}$ Max-Planck-Institut für Astrophysik, Karl-Schwarzschild-Str. 1, D-85741 Garching, Germany \\ ${ }^{8}$ INAF-Osservatorio Astronomico di Capodimonte, Salita Moiariello 16, I-80131 Napoli, Italy \\ ${ }^{9}$ Coral Towers Observatory, Coral Towers, Esplanade, Cairns 4870, Australia \\ ${ }^{10}$ Laboratoire de Physique Nucléaire et de Hautes Énergies, Université Pierre et Marie Curie Paris 6, Université Paris Diderot, Paris 7 , \\ CNRS-IN2P3, 4 place Jussieu, F-75252 Paris Cedex 05, France \\ ${ }^{11}$ Instituto de Astrofísica de Andalucía, CSIC, Apdo 3004, E-18080 Granada, Spain \\ 12 INAF-Osservatorio Astronomico di Trieste, via Tiepolo 11, I-34143 Trieste, Italy \\ ${ }^{13} 2$ Yandra Street, Vale Park, Adelaide, South Australia 5081, Australia \\ ${ }^{14}$ Institut de Ciencies de l'Espai (IEEC-CSIC), Campus UAB, E-08193 Bellaterra, Spain \\ 15 The Oskar Klein Centre, Department of Astronomy, AlbaNova, Stockholm University, SE-106 91 Stockholm, Sweden \\ ${ }^{16}$ Dark Cosmology Centre, Niels Bohr Institute, Copenhagen University, Juliane Maries Vej 30, DK-2100 Copenhagen O, Denmark \\ ${ }^{17}$ Vereniging Voor Sterrenkunde, Oude Bleken 12, B-2400 Mol, Belgium \\ ${ }^{18}$ Departamento de Astronomía, Universidad de Chile, Casilla 36-D, Santiago, Chile \\ ${ }^{19}$ Telescopio Nazionale Galileo, Fundación Galileo Galilei-INAF, Rambla José Ana Fernández Pérez 7, E-38712 Breña Baja, TF, Spain \\ ${ }^{20}$ Skynet Robotic Telescope Network, Department of Physics and Astronomy, University of North Carolina, Chapel Hill, NC 27599-3255, USA \\ ${ }^{21}$ Tuorla Observatory, Department of Physics and Astronomy, University of Turku, FI-21500 Piikkiö, Finland \\ ${ }^{22}$ Astronomy/Physics MS HSB 314, One University Plaza Springfield, IL 62730, USA \\ ${ }^{23}$ Department of Physics (Astrophysics), University of Oxford, DWB, Keble Road, Oxford OX1 3RH, UK \\ ${ }^{24}$ Finnish Centre for Astronomy with ESO (FINCA), University of Turku, Väisäläntie 20, FI-21500 Piikkiö, Finland \\ ${ }^{25}$ Department of Astronomy, Padova University, Vicolo dell'Osservatorio 3, I-35122 Padova, Italy \\ 26115 Adelma Road, Dalkeith, Western Australia 6009, Australia \\ Received 2012 October 12; accepted 2013 January 22; published 2013 March 18
}

\begin{abstract}
We report the results of a three-year-long dedicated monitoring campaign of a restless luminous blue variable (LBV) in NGC 7259. The object, named SN 2009ip, was observed photometrically and spectroscopically in the optical and near-infrared domains. We monitored a number of erupting episodes in the past few years, and increased the density of our observations during eruptive episodes. In this paper, we present the full historical data set from 2009 to 2012 with multi-wavelength dense coverage of the two high-luminosity events between 2012 August and September. We construct bolometric light curves and measure the total luminosities of these eruptive or explosive events. We label them the $2012 \mathrm{a}$ event (lasting $\sim 50$ days) with a peak of $3 \times 10^{41} \mathrm{erg} \mathrm{s}^{-1}$, and the $2012 \mathrm{~b}$ event (14 day rise time, still ongoing) with a peak of $8 \times 10^{42} \mathrm{erg} \mathrm{s}^{-1}$. The latter event reached an absolute $R$-band magnitude of about -18 , comparable to that of a core-collapse supernova (SN). Our historical monitoring has detected high-velocity spectral features $\left(\sim 13,000 \mathrm{~km} \mathrm{~s}^{-1}\right)$ in 2011 September, one year before the current SN-like event. This implies that the detection of such high-velocity outflows cannot, conclusively, point to a core-collapse SN origin. We suggest that the initial peak in the 2012a event was unlikely to be due to a faint core-collapse SN. We propose that the high intrinsic luminosity of the latest peak, the variability history of SN 2009ip, and the detection of broad spectral lines indicative of high-velocity ejecta are consistent with a pulsational pair-instability event, and that the star may have survived the last outburst. The question of the survival of the LBV progenitor star and its future fate remain open issues, only to be answered with future monitoring of this historically unique explosion.
\end{abstract}

Key words: galaxies: individual (NGC 7259) - supernovae: general - supernovae: individual (SN 2009ip, SN 2000ch)

Online-only material: color figures

\section{INTRODUCTION}

Luminous blue variables (LBVs) are among the most luminous and massive stars found in late-type galaxies. In a few cases, these stars have been observed to produce major eruptions that mimic a genuine supernova ( $\mathrm{SN}$ ) explosion. For this reason, they gained the label of SN impostors (Van Dyk et al. 2000). The discrimination between SN impostors (i.e., LBV-type eruptions) and Type IIn $\mathrm{SNe}$ can be ambiguous (see, e.g., the SN 2011ht-like objects; Roming et al. 2012; 
Mauerhan et al. 2012b; Humphreys et al. 2012; Kankare et al. 2012; Dessart et al. 2009; Chugai et al. 2004).

LBVs are observed in the Milky Way, Local Group galaxies, and beyond (e.g., Humphreys \& Davidson 1994; Humphreys et al. 1999; Maund et al. 2006; Smith et al. 2011b). They have high mass-loss rates and frequently show what is known as S Doradus variability during which mass loss is enhanced, possibly due to temperature changes and ionization balance of atomic species that drive the wind (Vink \& de Koter 2002). Giant eruptions have been observed during which several solar masses of material can be ejected (e.g., Davidson \& Humphreys 1997), and the intrinsic stellar luminosity increases substantially. The physical mechanism that triggers these giant eruptions is still unknown. Based on the analysis of SN data, a link between some LBVs and SNe IIn has been proposed (see, e.g., Kotak \& Vink 2006; Smith \& Owocki 2006; Smith et al. 2007; Trundle et al. 2008, 2009). There is at least one case (SN 2005gl) in which a likely LBV has been observed to explode as luminous SNe IIn (Gal-Yam et al. 2007; Gal-Yam \& Leonard 2009), and one other case (SN 2010jl) for which there is a plausible argument for a massive progenitor star of a Type IIn SN $\left(M>30 M_{\odot}\right.$; Smith et al. 2011a). ${ }^{27}$ In this context, a remarkable object is SN $1961 \mathrm{~V}$ in NGC 1058, whose nature (SN IIn versus LBV-type eruption) is still controversial (see Chu et al. 2004; Kochanek et al. 2011; Van Dyk \& Matheson 2012, and references therein).

In an exciting turn of events, a well-observed LBV in the spiral galaxy NGC 7259 (designated as SN 2009ip during a giant outburst in 2009) has recently been proposed to have finally exploded as a core-collapse SN (Mauerhan et al. 2012a, and references therein). The object was first discovered on 2009 August 26 by the CHASE SN Search (Maza et al. 2009) as a faint transient at $\approx 17.9 \mathrm{mag}$, and was later classified as an SN impostor by a number of teams (Miller et al. 2009; Li et al. 2009; Berger et al. 2009). The nature of SN 2009ip was widely discussed in Smith et al. (2010, 2011b) and Foley et al. (2011). Through the analysis of pre-outburst archival Hubble Space Telescope (HST) images these studies provided robust evidence that the progenitor was a very massive star that experienced repeated eruptions typical of the LBV phase. The main-sequence mass of the star estimated by Smith et al. (2010) was in the range 50-80 $M_{\odot}$, while Foley et al. (2011) found it to be $M_{\text {ZAMS }}>60 M_{\odot}$.

Subsequent re-brightenings were announced by the Catalina Real-Time Survey team on 2010 October 1 (Drake et al. 2010) and, very recently, on 2012 July 24 (Drake et al. 2012), which were first labeled as new LBV-type outbursts (e.g., Foley et al. 2012). However, from the detection of high-velocity spectral features on 2012 September 15 and 16, Smith \& Mauerhan (2012a) first mentioned the possibility that SN 2009ip exploded as a real core-collapse SN. ${ }^{28}$ High-cadence optical imaging in the $R$ and $I$ bands monitoring the strong 2012 September rebrightening has been presented by Prieto et al. (2013). We also note that although no (Margutti et al. 2012a, 2012b; Chandra \&

\footnotetext{
27 We note that eruptive Wolf-Rayet stars, producing impostors with a luminosity similar to that of an LBV outburst, have later on been observed to explode as He-rich Ibn SNe (Pastorello et al. 2007, 2008a; Foley et al. 2007) or hybrid IIn/Ibn events (Pastorello et al. 2008b; Smith et al. 2012).

28 We note that after the Smith \& Mauerhan communication there has been a proliferation of electronic telegrams on this transient, with different interpretations on its nature- $\mathrm{SN}$ vs. SN impostor (Margutti et al. 2012a, 2012b; Martin et al. 2012a, 2012b; Brimacombe 2012; Smith \& Mauerhan 2012b; Leonard et al. 2012; Burgasser et al. 2012; Vinko et al. 2012; Prieto et al. 2012; Gall et al. 2012; Bohlsen 2012; Vinko 2012; Jha et al.

2012) - although it seems that most authors now favor the SN explosion scenario.
}

Soderberg 2012; Hancock et al. 2012) or marginal (Campana 2012; Margutti \& Soderberg 2012) X-ray and radio detections of SN 2009ip were initially reported, an X-ray brightening was announced later (Campana \& Margutti 2012).

In this paper, we present observations of the LBV known as SN 2009ip in NGC 7259 over a period of three years including the following. (1) Data showing the history of erratic variability starting from 2009 August, when the object closely resembled NGC 3432-LBV1 (aka SN 2000ch; Wagner et al. 2004; Pastorello et al. 2010), an SN impostor that experienced multiple energetic outbursts. Our data of SN 2009ip also include observations of repeated outbursts during the period 2011 May to October which have not been reported to date. (2) The recent evolution of the LBV as a putative SN.

\section{OBSERVATIONS}

Three years ago, after the first announcement of the discovery of a transient in NGC 7259 (Maza et al. 2009), we initiated an extensive spectroscopic and photometric monitoring campaign in the optical bands using a number of telescopes available to our collaboration. After about 100 days, the follow-up strategy was relaxed and the photometric monitoring was limited to the $R$ band. Due to its unpredictable behavior, we kept up a monitoring campaign of this object during the following three years.

After the recent re-brightening of SN 2009ip announced by the Catalina Real-Time Survey team on 2012 July 24 (Drake et al. 2012), we intensified our observing cadence and secured multi-color photometry and spectroscopy from the optical to the near-IR domains.

In addition, Swift optical and ultraviolet observations have been triggered (PIs: R. Margutti and P. W. A. Roming) and included in our analysis, particularly to derive a pseudobolometric light curve of the 2012 eruptions.

\subsection{Photometry}

Photometric observations were carried on using a long list of facilities, namely, the $8.2 \mathrm{~m}$ Very Large Telescope (VLT) of the European Southern Observatory (ESO) equipped with FORS2 (Cerro Paranal, Chile), the 3.58 m ESO New Technology Telescope (NTT) equipped with EFOSC 2 and SOFI (La Silla, Chile), the $3.58 \mathrm{~m}$ Telescopio Nazionale Galileo (TNG) + LRS, the $2 \mathrm{~m}$ Liverpool Telescope with RATCam and the $2.56 \mathrm{~m}$ Nordic Optical Telescope (NOT) + ALFOSC and NOTCam (La Palma, Canary Islands, Spain); the $2 \mathrm{~m}$ Faulkes Telescope South + EM02 (Siding Spring Observatory, Australia); the $0.41 \mathrm{~m}$ Panchromatic Robotic Optical Monitoring and Polarimetry Telescopes (Cerro Tololo, Chile); and a group of 0.3 to $0.5 \mathrm{~m}$ telescopes in Australia and New Mexico, USA (see Table 1 for details). As mentioned above, the Swift satellite plus UVOT secured additional optical and ultraviolet photometry.

The pre-reduction of the optical photometry images was performed using standard IRAF $^{29}$ tasks, and these included bias, overscan, and flat-field corrections.

The pre-reduction of the NIR photometry images required a few additional steps, since we had to remove from the science images the contribution of the bright NIR sky. Clear sky images were therefore obtained by median-combining a number of dithered science frames and were then subtracted from the target images. Thereafter, the sky-subtracted science images

\footnotetext{
29 IRAF is distributed by the National Optical Astronomy Observatory, which is operated by the Association of Universities for Research in Astronomy (AURA) under cooperative agreement with the National Science Foundation.
} 
Table 1

Optical Photometry of SN 2009ip

\begin{tabular}{|c|c|c|c|c|c|c|c|}
\hline Date (dd/mm/yy) & $\mathrm{JD}-2,400,000$ & $U$ & $B$ & V & $R$ & $I$ & Source \\
\hline $30 / 08 / 09$ & 55074.14 & $\ldots$ & $\ldots$ & $\ldots$ & $\ldots$ & $18.11(0.13)$ & 1 \\
\hline $30 / 08 / 09$ & 55074.10 & $\ldots$ & $\ldots$ & $\ldots$ & $18.13(0.09)$ & $\ldots$ & 2 \\
\hline $30 / 08 / 09$ & 55074.24 & $\ldots$ & $\ldots$ & $18.51(0.18)$ & $\ldots$ & $\ldots$ & 2 \\
\hline 06/09/09 & 55080.92 & $\ldots$ & $\ldots$ & $20.99(0.37)$ & $\ldots$ & $\ldots$ & 2 \\
\hline 07/09/09 & 55081.56 & $\ldots$ & $\ldots$ & $21.06(0.05)$ & $20.43(0.03)$ & $\ldots$ & 3 \\
\hline $07 / 09 / 09$ & 55082.00 & $\ldots$ & $\ldots$ & $20.70(0.17)$ & $\ldots$ & $\ldots$ & 2 \\
\hline $07 / 09 / 09$ & 55082.02 & $\ldots$ & $\ldots$ & $20.73(0.20)$ & $\ldots$ & $\ldots$ & 2 \\
\hline 07/09/09 & 55082.03 & $\ldots$ & $\ldots$ & $\ldots$ & $19.78(0.24)$ & $\ldots$ & 1 \\
\hline 08/09/09 & 55083.43 & $\ldots$ & $\ldots$ & $\ldots$ & $20.17(0.08)$ & $\ldots$ & 4 \\
\hline 09/09/09 & 55083.80 & $\ldots$ & $\ldots$ & $20.72(0.32)$ & $\ldots$ & $\ldots$ & 5 \\
\hline 09/09/09 & 55084.03 & $\ldots$ & $\ldots$ & $20.82(0.25)$ & $\ldots$ & $\ldots$ & 2 \\
\hline 09/09/09 & 55084.06 & $\ldots$ & $\ldots$ & $\ldots$ & $20.18(0.20)$ & $\ldots$ & 1 \\
\hline $10 / 09 / 09$ & 55084.99 & $\ldots$ & $\ldots$ & $20.96(0.27)$ & $\ldots$ & $\ldots$ & 6 \\
\hline $10 / 09 / 09$ & 55085.42 & $\ldots$ & $\ldots$ & $\ldots$ & $20.66(0.07)$ & $\ldots$ & 4 \\
\hline $12 / 09 / 09$ & 55087.54 & $\ldots$ & $\ldots$ & $21.01(0.10)$ & $20.65(0.09)$ & $20.48(0.19)$ & 7 \\
\hline $13 / 09 / 09$ & 55087.92 & $\ldots$ & $\ldots$ & $\ldots$ & $\ldots$ & $>19.61$ & 1 \\
\hline $13 / 09 / 09$ & 55088.10 & $\ldots$ & $\ldots$ & $21.01(0.30)$ & $\ldots$ & $\ldots$ & 6 \\
\hline $14 / 09 / 09$ & 55088.81 & $\ldots$ & $\ldots$ & $\ldots$ & $\ldots$ & $>19.78$ & 5 \\
\hline $14 / 09 / 09$ & 55088.98 & $\ldots$ & $\ldots$ & $\ldots$ & $\ldots$ & $20.44(0.30)$ & 1 \\
\hline $14 / 09 / 09$ & 55089.42 & $\ldots$ & $\ldots$ & $\ldots$ & $20.61(0.06)$ & $\ldots$ & 4 \\
\hline $14 / 09 / 09$ & 55089.53 & $\ldots$ & $\ldots$ & $21.04(0.03)$ & $20.62(0.05)$ & $20.40(0.19)$ & 7 \\
\hline $23 / 09 / 09$ & 55098.39 & $\ldots$ & $\ldots$ & & $18.48(0.06)$ & $\ldots$ & 4 \\
\hline $24 / 09 / 09$ & 55098.72 & $\ldots$ & $\ldots$ & $18.95(0.13)$ & $\ldots$ & $\ldots$ & 5 \\
\hline $24 / 09 / 09$ & 55098.78 & $\ldots$ & $\ldots$ & $\ldots$ & $\ldots$ & $18.78(0.21)$ & 5 \\
\hline $24 / 09 / 09$ & 55099.42 & $\ldots$ & $19.64(0.03)$ & $19.32(0.04)$ & $19.01(0.04)$ & $18.97(0.10)$ & 4 \\
\hline $25 / 09 / 09$ & 55099.56 & $\ldots$ & $\ldots$ & $19.33(0.02)$ & $18.93(0.06)$ & $\ldots$ & 3 \\
\hline $25 / 09 / 09$ & 55099.65 & $\ldots$ & $\ldots$ & $19.39(0.12)$ & $\ldots$ & $\ldots$ & 5 \\
\hline $26 / 09 / 09$ & 55101.39 & $\ldots$ & $20.41(0.11)$ & $20.12(0.13)$ & $19.89(0.07)$ & $19.71(0.16)$ & 4 \\
\hline $27 / 09 / 09$ & 55101.73 & $\ldots$ & $\ldots$ & $20.11(0.25)$ & $\ldots$ & $\ldots$ & 5 \\
\hline 29/09/09 & 55103.64 & $\ldots$ & $\ldots$ & $19.67(0.03)$ & $19.32(0.03)$ & $\ldots$ & 3 \\
\hline 02/10/09 & 55107.37 & $\ldots$ & $\ldots$ & $\ldots$ & $20.04(0.14)$ & $\ldots$ & 4 \\
\hline $06 / 10 / 09$ & 55111.36 & $\ldots$ & $\ldots$ & $\ldots$ & $20.47(0.13)$ & $\ldots$ & 4 \\
\hline 08/10/09 & 55113.37 & $\ldots$ & $\ldots$ & $\ldots$ & $20.54(0.05)$ & $\ldots$ & 4 \\
\hline $10 / 10 / 09$ & 55114.91 & $\ldots$ & $\ldots$ & $21.26(0.36)$ & & $\ldots$ & 6 \\
\hline $13 / 10 / 09$ & 55118.46 & $\ldots$ & $\ldots$ & $21.21(0.10)$ & $20.51(0.06)$ & $20.39(0.10)$ & 4 \\
\hline $18 / 10 / 09$ & 55122.91 & $\ldots$ & $\ldots$ & $20.57(0.31)$ & $\ldots$ & $\ldots$ & 6 \\
\hline $22 / 10 / 09$ & 55126.60 & $\ldots$ & $20.91(0.03)$ & $20.76(0.09)$ & $20.38(0.17)$ & $20.31(0.16)$ & 7 \\
\hline $30 / 10 / 09$ & 55134.83 & $\ldots$ & $\ldots$ & $\ldots$ & $19.31(0.04)$ & $\ldots$ & 4 \\
\hline $02 / 11 / 09$ & 55137.87 & $\ldots$ & $\ldots$ & $\ldots$ & $20.09(0.06)$ & $\ldots$ & 4 \\
\hline $06 / 11 / 09$ & 55141.94 & $\ldots$ & $\ldots$ & $21.04(0.28)$ & $\ldots$ & $\ldots$ & 6 \\
\hline $07 / 11 / 09$ & 55142.93 & $\ldots$ & $\ldots$ & $20.91(0.18)$ & $\ldots$ & $\ldots$ & 6 \\
\hline $10 / 11 / 09$ & 55145.82 & $\ldots$ & $\ldots$ & $\ldots$ & $20.18(0.03)$ & $\ldots$ & 4 \\
\hline $13 / 11 / 09$ & 55148.83 & $\ldots$ & $\ldots$ & $\ldots$ & $19.77(0.02)$ & $\ldots$ & 4 \\
\hline $21 / 11 / 09$ & 55156.82 & $\ldots$ & $\ldots$ & $\ldots$ & $20.69(0.06)$ & $\ldots$ & 4 \\
\hline $22 / 11 / 09$ & 55157.55 & $\ldots$ & $\ldots$ & $\ldots$ & $21.06(0.04)$ & $\ldots$ & 7 \\
\hline $24 / 11 / 09$ & 55159.60 & $\ldots$ & $21.75(0.06)$ & $21.46(0.10)$ & $20.89(0.09)$ & $20.96(0.16)$ & 7 \\
\hline $29 / 11 / 09$ & 55163.83 & $\ldots$ & $\ldots$ & $\ldots$ & $20.59(0.09)$ & $\ldots$ & 4 \\
\hline $05 / 12 / 09$ & 55169.83 & $\ldots$ & $\ldots$ & $\ldots$ & $20.95(0.07)$ & $\ldots$ & 4 \\
\hline $04 / 10 / 10$ & 55473.79 & $\ldots$ & $\ldots$ & $\ldots$ & $20.29(0.15)$ & $\ldots$ & 7 \\
\hline $06 / 10 / 10$ & 55475.60 & $\ldots$ & $\ldots$ & $\ldots$ & $20.67(0.07)$ & $\ldots$ & 7 \\
\hline $06 / 10 / 10$ & 55475.63 & $\ldots$ & $\ldots$ & $\ldots$ & $20.65(0.03)$ & $\ldots$ & 7 \\
\hline $06 / 10 / 10$ & 55475.70 & $21.36(0.06)$ & $21.66(0.02)$ & $21.29(0.08)$ & $20.65(0.08)$ & $20.63(0.12)$ & 7 \\
\hline $28 / 10 / 10$ & 55497.67 & $\ldots$ & $\ldots$ & $\ldots$ & $19.92(0.03)$ & $\ldots$ & 7 \\
\hline $30 / 10 / 10$ & 55499.56 & $\ldots$ & $20.57(0.02)$ & $20.25(0.05)$ & $19.88(0.06)$ & $\ldots$ & 7 \\
\hline $30 / 10 / 10$ & 55499.63 & $\ldots$ & $\ldots$ & $\ldots$ & $\ldots$ & $19.80(0.12)$ & 7 \\
\hline $20 / 12 / 10$ & 55550.95 & $\ldots$ & $\ldots$ & $\ldots$ & $19.77(0.10)$ & $\ldots$ & 8 \\
\hline $23 / 12 / 10$ & 55553.95 & $\ldots$ & $\ldots$ & $\ldots$ & $20.14(0.16)$ & $\ldots$ & 8 \\
\hline $31 / 12 / 10$ & 55561.55 & $\ldots$ & $\ldots$ & $\ldots$ & $19.96(0.12)$ & $\ldots$ & 7 \\
\hline $02 / 01 / 11$ & 55563.55 & $\ldots$ & $\ldots$ & $\ldots$ & $19.93(0.08)$ & $\ldots$ & 7 \\
\hline $25 / 03 / 11$ & 55645.91 & $\cdots$ & $\cdots$ & $\cdots$ & $20.16(0.40)$ & $\cdots$ & 7 \\
\hline $11 / 04 / 11$ & 55662.90 & $\ldots$ & $\cdots$ & $\cdots$ & $20.87(0.20)$ & $\cdots$ & 7 \\
\hline $10 / 05 / 11$ & 55691.89 & $\ldots$ & $\ldots$ & $\ldots$ & $18.50(0.07)$ & $\ldots$ & 7 \\
\hline $25 / 05 / 11$ & 55707.13 & $\ldots$ & $\ldots$ & $\ldots$ & $20.59(0.15)$ & $\ldots$ & 8 \\
\hline $26 / 05 / 11$ & 55708.20 & $\ldots$ & $\ldots$ & $\ldots$ & $20.83(0.11)$ & $\ldots$ & 8 \\
\hline
\end{tabular}


Table 1

(Continued)

\begin{tabular}{|c|c|c|c|c|c|c|c|}
\hline Date (dd/mm/yy) & $\mathrm{JD}-2,400,000$ & $U$ & $B$ & $V$ & $R$ & $I$ & Source \\
\hline $27 / 05 / 11$ & 55709.19 & $\ldots$ & $\ldots$ & $\ldots$ & $20.81(0.18)$ & $\ldots$ & 8 \\
\hline $04 / 06 / 11$ & 55717.27 & $\ldots$ & $\ldots$ & $\ldots$ & $18.18(0.07)$ & $\ldots$ & 8 \\
\hline $10 / 06 / 11$ & 55723.26 & $\ldots$ & $\ldots$ & $\ldots$ & $20.12(0.17)$ & $\ldots$ & 8 \\
\hline $19 / 06 / 11$ & 55732.22 & $\ldots$ & $\ldots$ & $\ldots$ & $20.48(0.22)$ & $\ldots$ & 8 \\
\hline $24 / 06 / 11$ & 55736.82 & $\ldots$ & $\ldots$ & $\ldots$ & $20.63(0.10)$ & $\ldots$ & 7 \\
\hline $24 / 06 / 11$ & 55737.17 & $\ldots$ & $\ldots$ & $\ldots$ & $20.60(0.07)$ & $\ldots$ & 8 \\
\hline $26 / 06 / 11$ & 55738.78 & $\ldots$ & $\ldots$ & $\ldots$ & $20.87(0.09)$ & $\ldots$ & 7 \\
\hline $03 / 07 / 11$ & 55746.06 & $\ldots$ & $\ldots$ & $\ldots$ & $20.48(0.16)$ & $\ldots$ & 8 \\
\hline 08/07/11 & 55751.11 & $\ldots$ & $\ldots$ & $\ldots$ & $18.18(0.05)$ & $\ldots$ & 8 \\
\hline $14 / 07 / 11$ & 55757.02 & $\ldots$ & $\ldots$ & $\ldots$ & $20.03(0.41)$ & $\ldots$ & 8 \\
\hline $23 / 07 / 11$ & 55766.20 & $\ldots$ & $\ldots$ & $\ldots$ & $20.34(0.09)$ & $\ldots$ & 8 \\
\hline $28 / 07 / 11$ & 55771.21 & $\ldots$ & $\ldots$ & $\ldots$ & $20.45(0.08)$ & $\ldots$ & 8 \\
\hline 03/08/11 & 55777.24 & $\ldots$ & $\ldots$ & $\ldots$ & $18.46(0.05)$ & $\ldots$ & 8 \\
\hline $24 / 08 / 11$ & 55797.93 & $\ldots$ & $\ldots$ & $\ldots$ & $19.40(0.05)$ & $\ldots$ & 8 \\
\hline $27 / 08 / 11$ & 55801.12 & $\ldots$ & $\ldots$ & $\ldots$ & $19.91(0.03)$ & $\ldots$ & 8 \\
\hline $28 / 08 / 11$ & 55802.13 & $\ldots$ & $\ldots$ & $\ldots$ & $20.17(0.05)$ & $\ldots$ & 8 \\
\hline $30 / 08 / 11$ & 55804.24 & $\ldots$ & $\ldots$ & $\ldots$ & $20.39(0.05)$ & $\ldots$ & 8 \\
\hline $01 / 09 / 11$ & 55805.90 & $\ldots$ & $\ldots$ & $\ldots$ & $20.49(0.14)$ & $\ldots$ & 8 \\
\hline $03 / 09 / 11$ & 55808.01 & $\ldots$ & $\ldots$ & $\ldots$ & $20.73(0.07)$ & $\ldots$ & 8 \\
\hline $05 / 09 / 11$ & 55809.90 & $\ldots$ & $\ldots$ & $\ldots$ & $20.89(0.15)$ & $\ldots$ & 8 \\
\hline $06 / 06 / 11$ & 55810.66 & $\ldots$ & $\ldots$ & $\ldots$ & $21.00(0.08)$ & $\ldots$ & 7 \\
\hline $07 / 09 / 11$ & 55811.04 & $\ldots$ & $\ldots$ & $\ldots$ & $21.14(0.25)$ & $\ldots$ & 8 \\
\hline $10 / 09 / 11$ & 55815.02 & $\ldots$ & $\ldots$ & $\ldots$ & $>19.67$ & $\ldots$ & 8 \\
\hline $13 / 09 / 11$ & 55817.90 & $\ldots$ & $\ldots$ & $\ldots$ & $20.77(0.17)$ & $\ldots$ & 8 \\
\hline $16 / 09 / 11$ & 55821.18 & $\ldots$ & $\ldots$ & $\ldots$ & $20.29(0.11)$ & $\ldots$ & 8 \\
\hline $17 / 09 / 11$ & 55821.92 & $\ldots$ & $\ldots$ & $\ldots$ & $19.56(0.05)$ & $\ldots$ & 8 \\
\hline $19 / 09 / 11$ & 55823.94 & $\ldots$ & $\ldots$ & $\ldots$ & $18.57(0.03)$ & $\ldots$ & 8 \\
\hline $21 / 09 / 11$ & 55826.15 & $\ldots$ & $\ldots$ & $\ldots$ & $17.86(0.04)$ & $\ldots$ & 8 \\
\hline $22 / 09 / 11$ & 55826.91 & $\ldots$ & $\ldots$ & $\ldots$ & $18.48(0.04)$ & $\ldots$ & 8 \\
\hline $22 / 09 / 11$ & 55826.98 & $\ldots$ & $\ldots$ & $\ldots$ & $18.58(0.03)$ & $\ldots$ & 8 \\
\hline $22 / 09 / 11$ & 55827.09 & $\ldots$ & $\ldots$ & $\ldots$ & $18.72(0.03)$ & $\ldots$ & 8 \\
\hline $24 / 09 / 11$ & 55829.18 & $\ldots$ & $\ldots$ & $\ldots$ & $19.02(0.24)$ & $\ldots$ & 8 \\
\hline $26 / 09 / 11$ & 55830.90 & $\ldots$ & $\ldots$ & $\ldots$ & $19.36(0.07)$ & $\ldots$ & 8 \\
\hline $26 / 09 / 11$ & 55830.98 & $\ldots$ & $\ldots$ & $\ldots$ & $19.40(0.03)$ & $\ldots$ & 8 \\
\hline $26 / 09 / 11$ & 55831.13 & $\ldots$ & $\ldots$ & $\ldots$ & $19.45(0.06)$ & $\ldots$ & 8 \\
\hline $27 / 09 / 11$ & 55831.99 & $\ldots$ & $\ldots$ & $\ldots$ & $19.65(0.07)$ & $\ldots$ & 8 \\
\hline $30 / 09 / 11$ & 55834.90 & $\ldots$ & $\ldots$ & $\ldots$ & $20.02(0.05)$ & $\ldots$ & 8 \\
\hline $04 / 10 / 11$ & 55838.91 & $\ldots$ & $\ldots$ & $\ldots$ & $18.85(0.04)$ & $\ldots$ & 8 \\
\hline $09 / 10 / 11$ & 55843.90 & $\ldots$ & $\ldots$ & $\ldots$ & $>18.45$ & $\ldots$ & 8 \\
\hline $18 / 10 / 11$ & 55852.52 & $\ldots$ & $\ldots$ & $\ldots$ & $20.87(0.09)$ & $\ldots$ & 7 \\
\hline $18 / 10 / 11$ & 55852.99 & $\ldots$ & $\ldots$ & $\ldots$ & $20.82(0.08)$ & $\ldots$ & 8 \\
\hline $20 / 10 / 11$ & 55854.59 & $\ldots$ & $\ldots$ & $\ldots$ & $20.90(0.14)$ & $\ldots$ & 7 \\
\hline $21 / 10 / 11$ & 55855.91 & $\ldots$ & $\ldots$ & $\ldots$ & $20.84(0.07)$ & $\ldots$ & 8 \\
\hline $22 / 10 / 11$ & 55856.02 & $\ldots$ & $\ldots$ & $\ldots$ & $20.84(0.08)$ & $\ldots$ & 8 \\
\hline $24 / 10 / 11$ & 55858.91 & $\ldots$ & $\ldots$ & $\ldots$ & $20.50(0.06)$ & $\ldots$ & 8 \\
\hline $17 / 11 / 11$ & 55882.57 & $\ldots$ & $\ldots$ & $\ldots$ & $20.77(0.10)$ & $\ldots$ & 7 \\
\hline $27 / 11 / 11$ & 55892.94 & $\ldots$ & $\ldots$ & $\ldots$ & $19.96(0.06)$ & $\ldots$ & 8 \\
\hline $19 / 12 / 11$ & 55914.54 & $\ldots$ & $\ldots$ & $\ldots$ & $19.85(0.09)$ & $\ldots$ & 7 \\
\hline $21 / 12 / 11$ & 55916.53 & $\ldots$ & $\ldots$ & $\ldots$ & $19.70(0.11)$ & $\ldots$ & 7 \\
\hline $23 / 04 / 12$ & 56040.43 & $\ldots$ & $\ldots$ & $\ldots$ & $19.92(0.13)$ & $\ldots$ & 7 \\
\hline $08 / 08 / 12$ & 56147.73 & $17.54(0.01)$ & $18.51(0.01)$ & $18.43(0.04)$ & $18.22(0.03)$ & $18.12(0.05)$ & 7 \\
\hline $09 / 08 / 12$ & 56148.91 & $\ldots$ & $\ldots$ & $\ldots$ & $18.46(0.08)$ & $\ldots$ & 7 \\
\hline $11 / 08 / 12$ & 56150.90 & $\ldots$ & $\ldots$ & $\ldots$ & $18.23(0.05)$ & $\ldots$ & 7 \\
\hline $18 / 08 / 12$ & 56157.76 & $16.54(0.22)$ & $17.24(0.10)$ & $17.05(0.11)$ & $16.86(0.06)$ & $16.73(0.11)$ & 9 \\
\hline $25 / 08 / 12$ & 56164.78 & & $\ldots$ & $\ldots$ & $16.94(0.02)$ & & 7 \\
\hline $26 / 08 / 12$ & 56165.59 & $16.09(0.01)$ & $16.88(0.01)$ & $16.84(0.02)$ & $16.61(0.02)$ & $16.53(0.02)$ & 7 \\
\hline $27 / 08 / 12$ & 56166.65 & $16.24(0.01)$ & $17.02(0.01)$ & $16.93(0.01)$ & $16.73(0.03)$ & $16.58(0.03)$ & 7 \\
\hline $29 / 08 / 12$ & 56168.54 & $\ldots$ & $\ldots$ & - & $16.56(0.05)$ & $\ldots$ & 10 \\
\hline $31 / 08 / 12$ & 56170.54 & $16.32(0.02)$ & $17.02(0.01)$ & $16.84(0.02)$ & $16.54(0.05)$ & $16.48(0.08)$ & 10 \\
\hline $01 / 09 / 12$ & 56171.60 & $\ldots$ & $16.92(0.03)$ & $16.77(0.03)$ & $16.52(0.04)$ & $16.49(0.05)$ & 11 \\
\hline $02 / 09 / 12$ & 56172.62 & $\ldots$ & $17.02(0.08)$ & $16.73(0.02)$ & $16.57(0.04)$ & $16.41(0.04)$ & 11 \\
\hline $05 / 09 / 12$ & 56175.57 & $16.72(0.03)$ & $17.31(0.06)$ & $17.09(0.04)$ & $16.82(0.03)$ & $16.77(0.03)$ & 12 \\
\hline $05 / 09 / 12$ & 56175.85 & $\ldots$ & $\ldots$ & $16.99(0.07)$ & $16.78(0.07)$ & $16.79(0.10)$ & 11 \\
\hline $06 / 09 / 12$ & 56176.56 & $\ldots$ & $17.34(0.06)$ & $17.08(0.03)$ & $16.85(0.04)$ & $16.70(0.05)$ & 11 \\
\hline
\end{tabular}


Table 1

(Continued)

\begin{tabular}{|c|c|c|c|c|c|c|c|}
\hline Date (dd/mm/yy) & $\mathrm{JD}-2,400,000$ & $U$ & $B$ & V & $R$ & $I$ & Source \\
\hline $07 / 09 / 12$ & 56177.53 & 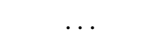 & $\ldots$ & $\ldots$ & $16.80(0.08)$ & $\ldots$ & 7 \\
\hline $07 / 09 / 12$ & 56177.60 & $16.39(0.12)$ & $\ldots$ & $\ldots$ & $\ldots$ & $\ldots$ & 7 \\
\hline $10 / 09 / 12$ & 56180.53 & $\ldots$ & $\ldots$ & $\ldots$ & $16.79(0.10)$ & $\ldots$ & 7 \\
\hline $11 / 09 / 12$ & 56181.67 & $\ldots$ & $\ldots$ & $16.98(0.05)$ & $16.58(0.04)$ & $16.57(0.07)$ & 13 \\
\hline $18 / 09 / 12$ & 56188.54 & $\ldots$ & $\ldots$ & $\ldots$ & $17.66(0.10)$ & & 7 \\
\hline $22 / 09 / 12$ & 56193.46 & $17.96(0.02)$ & $18.23(0.04)$ & $18.00(0.04)$ & $17.58(0.09)$ & $17.58(0.06)$ & 10 \\
\hline $23 / 09 / 12$ & 56193.59 & $\ldots$ & $18.22(0.07)$ & $17.97(0.04)$ & $17.61(0.06)$ & $17.48(0.07)$ & 11 \\
\hline $23 / 09 / 12$ & 56194.10 & $\ldots$ & $\ldots$ & $\ldots$ & $\ldots$ & $17.32(0.10)$ & 2 \\
\hline $24 / 09 / 12$ & 56194.58 & $\ldots$ & $18.14(0.06)$ & $17.84(0.03)$ & $17.53(0.06)$ & $17.45(0.06)$ & 11 \\
\hline $24 / 09 / 12$ & 56194.66 & $\ldots$ & $\ldots$ & $17.77(0.08)$ & $17.12(0.06)$ & $\ldots$ & 13 \\
\hline $24 / 09 / 12$ & 56195.09 & $\ldots$ & $\ldots$ & $\ldots$ & $16.32(0.05)$ & $16.53(0.07)$ & 2,6 \\
\hline $25 / 09 / 12$ & 56195.63 & $\ldots$ & $\ldots$ & $15.62(0.02)$ & $15.48(0.05)$ & $15.43(0.04)$ & 11 \\
\hline $25 / 09 / 12$ & 56195.70 & $\ldots$ & $\ldots$ & $\ldots$ & $\ldots$ & $15.03(0.12)$ & 5 \\
\hline $25 / 09 / 12$ & 56196.01 & $\ldots$ & $\ldots$ & $\ldots$ & $15.00(0.03)$ & $15.04(0.06)$ & 2,6 \\
\hline $26 / 09 / 12$ & 56196.57 & $\ldots$ & $\ldots$ & $14.91(0.06)$ & $\ldots$ & $\ldots$ & 14 \\
\hline $26 / 09 / 12$ & 56196.60 & $\ldots$ & $\ldots$ & $14.84(0.02)$ & $14.65(0.03)$ & $14.67(0.04)$ & 11 \\
\hline $26 / 09 / 12$ & 56196.98 & $\ldots$ & $\ldots$ & $\ldots$ & $14.52(0.05)$ & $14.56(0.05)$ & 2,6 \\
\hline $27 / 09 / 12$ & 56197.57 & $\ldots$ & $\ldots$ & $14.59(0.08)$ & $\ldots$ & $14.45(0.13)$ & 14 \\
\hline $27 / 09 / 12$ & 56197.63 & $\ldots$ & $\ldots$ & $14.52(0.02)$ & $14.37(0.03)$ & $14.39(0.04)$ & 11 \\
\hline $27 / 09 / 12$ & 56198.03 & $\ldots$ & $\ldots$ & $\ldots$ & $\ldots$ & $14.33(0.08)$ & 2 \\
\hline $28 / 09 / 12$ & 56198.56 & $\ldots$ & $\ldots$ & $14.39(0.06)$ & $\ldots$ & $14.19(0.07)$ & 14 \\
\hline $28 / 09 / 12$ & 56198.80 & $\ldots$ & $14.32(0.03)$ & $14.32(0.02)$ & $14.09(0.04)$ & $14.12(0.03)$ & 11 \\
\hline $28 / 09 / 12$ & 56198.93 & $\ldots$ & $\ldots$ & $\ldots$ & $14.14(0.05)$ & $14.21(0.07)$ & 2,6 \\
\hline $28 / 09 / 12$ & 56199.42 & $13.20(0.03)$ & $14.20(0.02)$ & $14.28(0.02)$ & $14.11(0.01)$ & $14.11(0.02)$ & 4 \\
\hline $29 / 09 / 12$ & 56199.65 & $\ldots$ & $14.28(0.02)$ & $14.20(0.01)$ & $14.09(0.02)$ & $14.08(0.02)$ & 11 \\
\hline $29 / 09 / 12$ & 56199.70 & $\ldots$ & $\ldots$ & $14.23(0.06)$ & $\ldots$ & $13.96(0.07)$ & 14 \\
\hline $29 / 09 / 12$ & 56200.08 & $\ldots$ & $\ldots$ & $\ldots$ & $14.05(0.02)$ & & 15 \\
\hline $29 / 09 / 12$ & 56200.43 & $13.03(0.03)$ & $14.09(0.02)$ & $14.15(0.02)$ & $13.98(0.01)$ & $13.98(0.02)$ & 4 \\
\hline $30 / 09 / 12$ & 56200.57 & $\ldots$ & $\ldots$ & $14.16(0.07)$ & $\ldots$ & $13.99(0.06)$ & 14 \\
\hline $30 / 09 / 12$ & 56201.11 & $\ldots$ & $\ldots$ & $\ldots$ & $14.08(0.02)$ & $\ldots$ & 15 \\
\hline $30 / 09 / 12$ & 56201.19 & $\ldots$ & $\ldots$ & $\ldots$ & $13.91(0.04)$ & $13.98(0.03)$ & 2,6 \\
\hline $01 / 10 / 12$ & 56201.53 & $\ldots$ & $14.02(0.04)$ & $14.03(0.02)$ & $13.91(0.02)$ & $13.95(0.01)$ & 11 \\
\hline $01 / 10 / 12$ & 56201.56 & $\ldots$ & $\ldots$ & $14.02(0.05)$ & $\ldots$ & $13.91(0.04)$ & 14 \\
\hline $01 / 10 / 12$ & 56201.94 & $\ldots$ & $\ldots$ & $\ldots$ & $13.85(0.03)$ & $13.94(0.03)$ & 2,6 \\
\hline $01 / 10 / 12$ & 56202.04 & $\ldots$ & $\ldots$ & $\ldots$ & $13.91(0.02)$ & $\ldots$ & 15 \\
\hline $01 / 10 / 12$ & 56202.44 & $12.98(0.03)$ & $13.97(0.02)$ & $14.03(0.02)$ & $13.86(0.01)$ & $13.88(0.02)$ & 4 \\
\hline $02 / 10 / 12$ & 56202.55 & $\ldots$ & $\ldots$ & $13.99(0.07)$ & $\ldots$ & $13.81(0.08)$ & 14 \\
\hline $02 / 10 / 12$ & 56202.76 & $\ldots$ & $\ldots$ & $13.96(0.05)$ & $\ldots$ & $\ldots$ & 5 \\
\hline $03 / 10 / 12$ & 56203.55 & $\ldots$ & $\ldots$ & $13.96(0.06)$ & $\ldots$ & $13.88(0.08)$ & 14 \\
\hline $03 / 10 / 12$ & 56203.72 & $\ldots$ & $\ldots$ & $13.86(0.03)$ & $\ldots$ & $\ldots$ & 5 \\
\hline $04 / 10 / 12$ & 56204.52 & $\ldots$ & $13.95(0.05)$ & $13.75(0.02)$ & $13.78(0.03)$ & $13.79(0.05)$ & 11 \\
\hline $04 / 10 / 12$ & 56204.55 & $\ldots$ & $\ldots$ & $13.90(0.05)$ & $\ldots$ & $13.80(0.07)$ & 14 \\
\hline $04 / 10 / 12$ & 56205.11 & $\ldots$ & $\ldots$ & $\ldots$ & $13.74(0.01)$ & & 15 \\
\hline $05 / 10 / 12$ & 56205.55 & $\ldots$ & $\ldots$ & $13.81(0.05)$ & $\ldots$ & $13.72(0.07)$ & 14 \\
\hline $05 / 10 / 12$ & 56205.61 & $\ldots$ & $\ldots$ & $13.73(0.05)$ & $\ldots$ & $\ldots$ & 5 \\
\hline $05 / 10 / 12$ & 56206.09 & $\ldots$ & $\ldots$ & & $13.70(0.01)$ & $\ldots$ & 15 \\
\hline $06 / 10 / 12$ & 56206.54 & $\ldots$ & $\ldots$ & $13.80(0.05)$ & $\ldots$ & $13.68(0.05)$ & 14 \\
\hline $06 / 10 / 12$ & 56206.71 & $\ldots$ & $\ldots$ & $13.71(0.03)$ & $\ldots$ & $\ldots$ & 5 \\
\hline $06 / 10 / 12$ & 56207.09 & $\ldots$ & $\ldots$ & & $13.70(0.01)$ & $\ldots$ & 15 \\
\hline $06 / 10 / 12$ & 56207.40 & $12.82(0.04)$ & $13.80(0.02)$ & $13.82(0.02)$ & $13.65(0.02)$ & $13.66(0.02)$ & 4 \\
\hline $07 / 10 / 12$ & 56208.41 & $\ldots$ & $\ldots$ & $13.83(0.02)$ & $13.65(0.01)$ & $13.67(0.02)$ & 4 \\
\hline $08 / 10 / 12$ & 56208.54 & $\ldots$ & $\ldots$ & $13.83(0.05)$ & $\ldots$ & $13.69(0.07)$ & 14 \\
\hline $08 / 10 / 12$ & 56209.42 & $\ldots$ & $\ldots$ & $13.88(0.02)$ & $13.73(0.01)$ & $13.68(0.02)$ & 4 \\
\hline $09 / 10 / 12$ & 56209.60 & $\ldots$ & $\ldots$ & $13.88(0.03)$ & $13.75(0.04)$ & $13.69(0.07)$ & 13 \\
\hline $09 / 10 / 12$ & 56209.70 & $\ldots$ & $\ldots$ & $13.86(0.05)$ & & $13.63(0.09)$ & 14 \\
\hline $09 / 10 / 12$ & 56210.07 & $\ldots$ & $\ldots$ & $\ldots$ & $13.74(0.01)$ & $\ldots$ & 15 \\
\hline $09 / 10 / 12$ & 56210.43 & $12.99(0.03)$ & $13.91(0.02)$ & $13.92(0.01)$ & $13.73(0.01)$ & $13.70(0.02)$ & 4 \\
\hline $10 / 10 / 12$ & 56210.53 & $\ldots$ & $\ldots$ & $13.87(0.06)$ & $\ldots$ & $13.71(0.07)$ & 14 \\
\hline $10 / 10 / 12$ & 56210.65 & $\ldots$ & $13.95(0.03)$ & $\ldots$ & $\ldots$ & $\ldots$ & 11 \\
\hline $10 / 10 / 12$ & 56211.08 & $\ldots$ & $\ldots$ & $\ldots$ & $13.76(0.01)$ & $\ldots$ & 15 \\
\hline $11 / 10 / 12$ & 56211.65 & $\ldots$ & $\ldots$ & $13.91(0.03)$ & & $13.61(0.07)$ & 14 \\
\hline $11 / 10 / 12$ & 56212.39 & $13.04(0.03)$ & $13.96(0.02)$ & $13.90(0.02)$ & $13.73(0.01)$ & $13.69(0.02)$ & 4 \\
\hline $12 / 10 / 12$ & 56212.53 & $\ldots$ & $\ldots$ & $13.87(0.06)$ & $\ldots$ & $13.67(0.06)$ & 14 \\
\hline $13 / 10 / 12$ & 56213.53 & $\ldots$ & $\ldots$ & $13.87(0.01)$ & $13.74(0.03)$ & $13.66(0.05)$ & 11 \\
\hline
\end{tabular}


Table 1

(Continued)

\begin{tabular}{|c|c|c|c|c|c|c|c|}
\hline Date $(\mathrm{dd} / \mathrm{mm} / \mathrm{yy})$ & JD-2,400,000 & $U$ & $B$ & $V$ & $R$ & $I$ & Source \\
\hline $13 / 10 / 12$ & 56213.58 & $\ldots$ & $\ldots$ & $13.86(0.06)$ & & $13.59(0.11)$ & 14 \\
\hline $13 / 10 / 12$ & 56214.38 & $13.02(0.03)$ & $13.96(0.02)$ & $13.91(0.02)$ & $13.71(0.01)$ & $13.68(0.02)$ & 4 \\
\hline $15 / 10 / 12$ & 56216.11 & $\ldots$ & $\ldots$ & $\ldots$ & $13.79(0.01)$ & $\ldots$ & 15 \\
\hline $16 / 10 / 12$ & 56216.73 & $\ldots$ & $14.11(0.02)$ & $14.00(0.03)$ & $13.79(0.02)$ & $13.74(0.01)$ & 11 \\
\hline
\end{tabular}

Notes.

${ }^{1} 0.3 \mathrm{~m}$ Mewlon Telescope + ST10 XME camera, Coral Tower Observatory, Cairns (Australia).

${ }^{2} 0.41 \mathrm{~m}$ RCOS Telescope + STL6K camera, Coral Tower Observatory, Cairns (Australia).

$38.2 \mathrm{~m}$ VLT UT1 + FORS2, ESO, Cerro Paranal (Chile).

${ }^{4} 2 \mathrm{~m}$ Liverpool Telescope + RATCam, La Palma, Canary Islands (Spain).

${ }^{5} 0.51 \mathrm{~m}$ RCOS Telescope + STL11K camera, New Mexico Skies, Mayhill, New Mexico (USA).

${ }^{6} 0.33$ m RCOS Telescope + STL6K camera, in 2009 at the Macedon Ranges Observatory, Melbourne; in 2012 at the Coral Tower Observatory,

Cairns (Australia).

73.58 m NTT + EFOSC2, ESO, La Silla (Chile).

$82 \mathrm{~m}$ Faukes Telescope South + EM03, Siding Spring Observatory (Australia).

$98.2 \mathrm{~m}$ VLT UT2 + XShooter (spectrophotometry), ESO, Cerro Paranal (Chile).

${ }^{10} 3.58 \mathrm{~m}$ TNG + Dolores, La Palma, Canary Islands (Spain).

${ }^{11} 0.41 \mathrm{~m}$ Panchromatic Robotic Optical Monitoring and Polarimetry Telescopes (PROMPT) (3+5), at Cerro Tololo Inter-American Observatory (Chile).

$122.56 \mathrm{~m}$ NOT + ALFOSC, La Palma, Canary Islands (Spain).

$130.51 \mathrm{~m}$ Cassegrain Reflector + Apogee U42 CCD Camera, Barber Observatory, Pleasant Plains, Illinois (USA).

${ }^{14} 0.40$ m Optimized Dall-Kirkham + FLI Kodak 16803 CCD, Remote Observatory Atacama Desert, San Pedro de Atacama (Chile).

150.235 m Perth Exoplanet Survey Telescope + SBIG ST-8XME CCD, Perth (Australia).

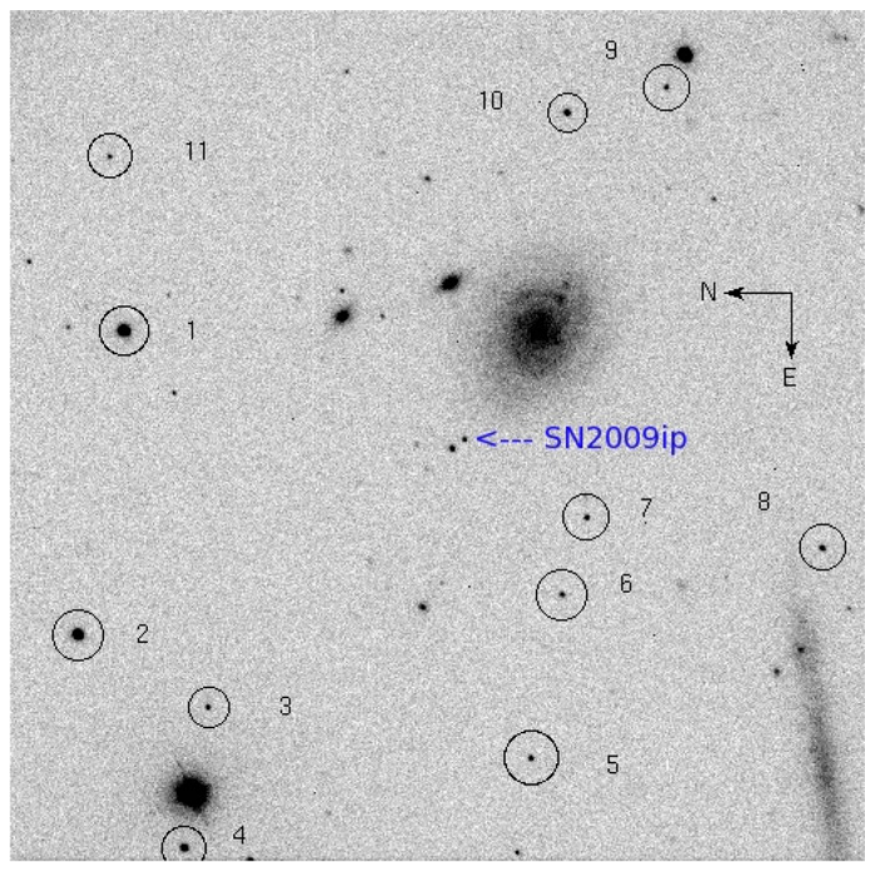

Figure 1. SN 2009ip in NGC 7259, and reference stars in the host galaxy field. (A color version of this figure is available in the online journal.)

were spatially registered and combined in order to improve the signal to noise.

SN 2009ip is located close to a red $(R=18.05 \pm 0.04, R-$ $I=0.72 \pm 0.05$ ) foreground star, in a remote position northeast of the host galaxy (Figure 1). Our optical and NIR photometric measurements were performed using the point-spread function fitting technique, with the simultaneous fit of the transient and the nearby star. A number of reference stars in the $\mathrm{SN}$ field were calibrated using observations of standard fields from the catalog of Landolt (1992), and used to improve the photometric calibration of the optical photometry of SN 2009ip in non- photometric nights. The NIR photometry of the reference stars was calibrated against the Two Micron All Sky Survey (2MASS) catalog magnitudes (Skrutskie et al. 2006).

Swift/UVOT data (in the uvw2, uvm 2, uvw1, $u, b$, and $v$ bands) were reduced using the HEASARC ${ }^{30}$ software. Images obtained on the same epoch were co-added, and finally reduced using the prescriptions of Poole et al. (2008).

The final photometry of the transient is reported in $\mathrm{Ta}-$ bles $1,{ }^{31} 2$, and 3 . Unpublished optical photometry of the comparison object NGC 3432-LBV1 is reported in Table 4. The magnitudes of the reference stars in the field of NGC 7259 are listed in Table 5.

The $R$-band absolute light curve of SN 2009ip starting from 2009 August and spanning a period of more than three years is shown in Figure 2 along with that of a similar event, NGC 3432-LBV1 (Pastorello et al. 2010), the debated transient (SN or impostor) 1961V (photographic magnitudes; Bertola 1963, 1964, 1965, 1967), and the revised visual light curve of the Giant Eruption of $\eta$ Carinae in 1842-1845 (see Smith et al. $2011 \mathrm{~b}$ and references therein). The same distance modulus $(\mu=$ $31.55 \pm 0.15 \mathrm{mag})$ and interstellar extinction $\left(A_{R}=0.051\right)$ adopted by Smith et al. (2010) and Mauerhan et al. (2012a) for SN 2009ip have been used in the absolute $R$-band light curve of Figure 2. The erratic light curves of all these transients show similar features. SN 2009ip experienced a few intense eruptive phases, including those on 2009 August-September and from 2011 May to October, characterized by a sequence of sharp luminosity peaks followed by rapid magnitude declines.

\footnotetext{
30 NASA's High Energy Astrophysics Science Archive Research Center. 31 We find excellent agreement with the CRTS and Prieto et al. (2013) photometry. The first version of Mauerhan et al. (2012a) posted to the arXiv archive showed a large disagreement with our photometry, being fainter by more than 2 mag in the $B$ band and 0.3 mag in the $I$ band. The $R$-band data were in reasonable agreement to the order of a few hundredths of a magnitude. Hence the average $B-R$ color computed with the Mauerhan et al. photometry was $\sim 2.5$, vs. $B-R \sim 0.5-0.7$ that is calculated with our data. However, subsequent versions of the Mauerhan et al. paper (versions 2 and 3) show fair agreement with our photometry, with differences of a few tenths of a magnitude.
} 
Table 2

Swift/UVOT Photometry of SN 2009ip

\begin{tabular}{|c|c|c|c|c|c|c|c|}
\hline Date $(\mathrm{dd} / \mathrm{mm} / \mathrm{yy})$ & JD & $U V w 2$ & $U V m 2$ & $U V w 1$ & $u$ & $b$ & $v$ \\
\hline $10 / 09 / 09$ & 55085.17 & $>21.64$ & $>21.10$ & $>21.18$ & $>20.87$ & $>21.22$ & $>20.30$ \\
\hline $04 / 09 / 12$ & 56175.40 & $18.00(0.08)$ & $\ldots$ & $17.20(0.05)$ & $16.49(0.04)$ & $17.29(0.04)$ & $17.01(0.02)$ \\
\hline $06 / 09 / 12$ & 56177.14 & $18.02(0.08)$ & $17.95(0.08)$ & $17.06(0.07)$ & $16.51(0.06)$ & $17.33(0.07)$ & $17.23(0.12)$ \\
\hline $13 / 09 / 12$ & 56183.94 & $19.38(0.32)$ & $19.04(0.16)$ & $18.29(0.12)$ & $17.31(0.07)$ & $17.73(0.08)$ & $17.83(0.14)$ \\
\hline $20 / 09 / 12$ & 56191.25 & $\ldots$ & $\ldots$ & $19.30(0.24)$ & $17.91(0.13)$ & $18.50(0.17)$ & $\ldots$ \\
\hline $22 / 09 / 12$ & 56193.26 & $20.58(0.26)$ & $20.52(0.32)$ & $19.36(0.23)$ & $17.91(0.07)$ & $18.24(0.06)$ & $18.17(0.07)$ \\
\hline $27 / 09 / 12$ & 56197.95 & $12.61(0.04)$ & $\ldots$ & $12.78(0.04)$ & $\ldots$ & $\ldots$ & $\ldots$ \\
\hline $27 / 09 / 12$ & 56198.20 & $12.50(0.04)$ & $\ldots$ & $12.68(0.04)$ & $13.02(0.04)$ & $14.40(0.04)$ & $\ldots$ \\
\hline $28 / 09 / 12$ & 56198.54 & $12.47(0.04)$ & $12.36(0.04)$ & $12.66(0.04)$ & $12.90(0.04)$ & $14.40(0.05)$ & $14.58(0.06)$ \\
\hline $28 / 09 / 12$ & 56199.09 & $12.39(0.04)$ & $\ldots$ & $12.63(0.04)$ & $\ldots$ & $\ldots$ & $\ldots$ \\
\hline $29 / 09 / 12$ & 56199.96 & $\ldots$ & $\ldots$ & $12.57(0.04)$ & $\ldots$ & $\ldots$ & $\ldots$ \\
\hline $29 / 09 / 12$ & 56200.15 & $12.28(0.04)$ & $12.38(0.05)$ & $12.45(0.04)$ & $12.81(0.04)$ & $14.11(0.04)$ & $14.22(0.04)$ \\
\hline $30 / 09 / 12$ & 56200.74 & $\ldots$ & $\ldots$ & $\ldots$ & $12.75(0.04)$ & $\ldots$ & $\ldots$ \\
\hline $30 / 09 / 12$ & 56201.49 & $12.18(0.04)$ & $12.07(0.04)$ & $12.35(0.04)$ & $12.67(0.04)$ & $13.94(0.04)$ & $14.12(0.04)$ \\
\hline $01 / 10 / 12$ & 56201.55 & $12.19(0.04)$ & $\ldots$ & $\ldots$ & $\ldots$ & $\ldots$ & $\ldots$ \\
\hline $01 / 10 / 12$ & 56201.93 & $12.20(0.04)$ & $\ldots$ & $\ldots$ & $\ldots$ & $\ldots$ & $\ldots$ \\
\hline $01 / 10 / 12$ & 56202.49 & $12.16(0.04)$ & $12.10(0.04)$ & $12.36(0.04)$ & $12.65(0.04)$ & $13.99(0.04)$ & $14.04(0.04)$ \\
\hline $02 / 10 / 12$ & 56202.76 & $12.18(0.04)$ & $12.11(0.04)$ & $12.36(0.04)$ & $12.65(0.04)$ & $13.95(0.04)$ & $14.08(0.04)$ \\
\hline $03 / 10 / 12$ & 56203.56 & $12.20(0.04)$ & $12.11(0.04)$ & $12.36(0.05)$ & $12.65(0.05)$ & $13.96(0.04)$ & $14.09(0.04)$ \\
\hline $03 / 10 / 12$ & 56203.79 & $12.23(0.04)$ & $\ldots$ & $\ldots$ & $\ldots$ & $\ldots$ & $\ldots$ \\
\hline $04 / 10 / 12$ & 56204.55 & $12.14(0.04)$ & $12.04(0.04)$ & $12.30(0.05)$ & $12.63(0.05)$ & $13.86(0.04)$ & $13.92(0.04)$ \\
\hline $04 / 10 / 12$ & 56204.89 & $12.10(0.04)$ & $\ldots$ & $\ldots$ & $\ldots$ & $\ldots$ & $\ldots$ \\
\hline $04 / 10 / 12$ & 56205.01 & $\ldots$ & $\ldots$ & $\ldots$ & $\ldots$ & $13.80(0.12)$ & $\ldots$ \\
\hline $05 / 10 / 12$ & 56205.90 & $12.10(0.04)$ & $\ldots$ & $\ldots$ & $\ldots$ & $\ldots$ & $\ldots$ \\
\hline $05 / 10 / 12$ & 56206.02 & $\ldots$ & $\ldots$ & $\ldots$ & $\ldots$ & $13.77(0.10)$ & $\ldots$ \\
\hline $06 / 10 / 12$ & 56206.62 & $12.10(0.04)$ & $12.02(0.04)$ & $12.24(0.04)$ & $12.52(0.05)$ & $13.75(0.05)$ & $13.86(0.04)$ \\
\hline $06 / 10 / 12$ & 56206.70 & $12.13(0.04)$ & $\ldots$ & $\ldots$ & $\ldots$ & $\ldots$ & $\ldots$ \\
\hline $07 / 10 / 12$ & 56207.93 & $12.27(0.04)$ & $\ldots$ & $\ldots$ & $\ldots$ & $\ldots$ & $\ldots$ \\
\hline $12 / 10 / 12$ & 56212.70 & $12.76(0.05)$ & $12.55(0.05)$ & $12.74(0.05)$ & $12.77(0.05)$ & $13.91(0.05)$ & $13.94(0.05)$ \\
\hline $14 / 10 / 12$ & 56214.63 & $12.90(0.05)$ & $12.68(0.05)$ & $12.82(0.05)$ & $12.86(0.05)$ & $13.98(0.05)$ & $13.93(0.05)$ \\
\hline $16 / 10 / 12$ & 56216.71 & $13.39(0.05)$ & $13.12(0.05)$ & $13.33(0.05)$ & $13.07(0.05)$ & $14.14(0.05)$ & $14.07(0.05)$ \\
\hline
\end{tabular}

Note. The photometry at the epoch marked with an asterisk has been published by Margutti et al. (2012a).

Table 3

Near-infrared Photometry of SN 2009ip Obtained During the 2012a and 2012b Events

\begin{tabular}{lccccc}
\hline \hline Date $(\mathrm{dd} / \mathrm{mm} / \mathrm{yy})$ & JD & $J$ & $H$ & $K s$ & Source \\
\hline $18 / 08 / 12$ & 56157.76 & $16.32(0.177)$ & $16.16(0.16)$ & $16.60(0.22)$ & 1 \\
$26 / 08 / 12$ & 56165.80 & $16.25(0.098)$ & $16.11(0.12)$ & $15.88(0.15)$ & 2 \\
$08 / 09 / 12$ & 56178.53 & $16.47(0.132)$ & $16.28(0.13)$ & $15.92(0.18)$ & 3 \\
$09 / 09 / 12$ & 56179.60 & $16.46(0.102)$ & $16.28(0.12)$ & $16.02(0.15)$ & 2 \\
$22 / 09 / 12$ & 56193.59 & $17.17(0.120)$ & $17.15(0.18)$ & $16.86(0.17)$ & 2 \\
$27 / 09 / 12$ & 56198.48 & $14.17(0.097)$ & $13.92(0.11)$ & $13.48(0.16)$ & 3 \\
$28 / 09 / 12$ & 56199.47 & $14.06(0.108)$ & $13.78(0.13)$ & $13.38(0.17)$ & 3 \\
$01 / 10 / 12$ & 56202.46 & $13.97(0.110)$ & $13.73(0.14)$ & $13.29(0.18)$ & 3 \\
$12 / 10 / 12$ & 56212.75 & $13.65(0.184)$ & $13.35(0.17)$ & $13.38(0.27)$ & 4 \\
\hline
\end{tabular}

Notes.

18.2 m VLT UT2 + XShooter (spectrophotometry), ESO, Cerro Paranal (Chile).

$23.58 \mathrm{~m} \mathrm{NTT}+$ SOFI, ESO, La Silla (Chile).

$32.56 \mathrm{~m}$ NOT + NOTCam, La Palma, Canary Islands (Spain).

${ }^{4} 0.6$ m Rapid Eye Mount + REMIR, La Silla (Chile).

The detailed, multi-band light curve of SN 2009ip during different outbursts is shown in Figure 3, with the 2009 event in the top panel (BVRI bands), the 2011 event in the middle panel ( $R$-band only), and the 2012 outbursts in the bottom panel (from the ultraviolet Swift data to the NIR bands).
The 2009 and 2011 eruptive phases present the erratic evolution typical of LBV-type giant eruption, and is very similar to those observed in the Giant Eruption of $\eta$ Carinae and in NGC 3432-LBV1. Other re-brightenings were registered by CRTS (to magnitudes $V \sim 17$ on 2010 July 15 , and $V \sim 17.7$ on 
Table 4

Unpublished Optical Photometry of NGC 3432-LBV1 (aka SN 2000ch)

\begin{tabular}{|c|c|c|c|c|c|c|c|}
\hline Date $(\mathrm{dd} / \mathrm{mm} / \mathrm{yy})$ & $\mathrm{JD}-2,400,000$ & $U$ & $B$ & $V$ & $R$ & $I$ & Source \\
\hline $02 / 07 / 10$ & 55380.37 & $\ldots$ & $\ldots$ & $\ldots$ & $19.78(0.05)$ & $\ldots$ & 1 \\
\hline $14 / 07 / 10$ & 55392.37 & $\ldots$ & $\ldots$ & $\ldots$ & $19.09(0.05)$ & $\ldots$ & 1 \\
\hline $20 / 07 / 10$ & 55398.36 & $\ldots$ & $\ldots$ & $\ldots$ & $20.53(0.31)$ & $\ldots$ & 1 \\
\hline $10 / 10 / 10$ & 55489.70 & $\ldots$ & $\ldots$ & $\ldots$ & $19.74(0.10)$ & $\ldots$ & 1 \\
\hline $26 / 10 / 10$ & 55495.66 & $\ldots$ & $22.25(0.40)$ & $20.97(0.17)$ & $19.99(0.06)$ & $20.17(0.22)$ & 1 \\
\hline $17 / 11 / 10$ & 55517.71 & $\ldots$ & $\ldots$ & $\ldots$ & $19.93(0.11)$ & $\ldots$ & 1 \\
\hline $20 / 11 / 10$ & 55520.65 & $\ldots$ & $\ldots$ & $\ldots$ & $20.38(0.09)$ & $\ldots$ & 1 \\
\hline $15 / 01 / 11$ & 55576.75 & $\ldots$ & $\ldots$ & $\ldots$ & $19.14(0.05)$ & $\ldots$ & 1 \\
\hline $08 / 02 / 11$ & 55600.70 & $\ldots$ & $\ldots$ & $\ldots$ & $19.98(0.07)$ & $\ldots$ & 2 \\
\hline $09 / 02 / 11$ & 55601.53 & $\ldots$ & $21.21(0.12)$ & $20.90(0.10)$ & $20.05(0.09)$ & $19.97(0.15)$ & 3 \\
\hline $09 / 02 / 11$ & 55602.49 & $\ldots$ & $\ldots$ & $\ldots$ & $20.09(0.05)$ & $\ldots$ & 3 \\
\hline $22 / 02 / 11$ & 55615.77 & $\ldots$ & $\ldots$ & $\ldots$ & $19.97(0.05)$ & $\ldots$ & 4 \\
\hline $06 / 04 / 11$ & 55658.49 & $\ldots$ & $21.26(0.15)$ & $21.25(0.19)$ & $20.28(0.18)$ & $>19.99$ & 3 \\
\hline $10 / 04 / 11$ & 55662.40 & $\ldots$ & $\ldots$ & $\ldots$ & $20.23(0.12)$ & $\ldots$ & 1 \\
\hline $01 / 05 / 11$ & 55683.40 & $\ldots$ & $\ldots$ & $\ldots$ & $>19.54$ & $\ldots$ & 3 \\
\hline $07 / 05 / 11$ & 55689.48 & $\ldots$ & $\ldots$ & $\ldots$ & $19.42(0.04)$ & $\ldots$ & 1 \\
\hline $22 / 05 / 11$ & 55704.35 & $\ldots$ & $\ldots$ & $\ldots$ & $19.17(0.05)$ & $\ldots$ & 1 \\
\hline $30 / 06 / 11$ & 55743.37 & $\ldots$ & $\ldots$ & $\ldots$ & $19.96(0.11)$ & $\ldots$ & 1 \\
\hline $01 / 11 / 11$ & 55866.56 & $>19.61$ & $>22.21$ & $>21.35$ & $20.48(0.23)$ & $>20.22$ & 3 \\
\hline $02 / 11 / 11$ & 55867.69 & $\ldots$ & $\ldots$ & $21.80(0.36)$ & $20.53(0.19)$ & $20.91(0.28)$ & 1 \\
\hline $16 / 11 / 11$ & 55881.72 & $\ldots$ & $\ldots$ & $\ldots$ & $20.95(0.33)$ & $\ldots$ & 1 \\
\hline $20 / 11 / 11$ & 55885.63 & $\ldots$ & $\ldots$ & $\ldots$ & $21.18(0.20)$ & $\ldots$ & 3 \\
\hline $22 / 12 / 11$ & 55917.62 & $\ldots$ & $\ldots$ & $\ldots$ & $21.07(0.07)$ & $\ldots$ & 2 \\
\hline $24 / 12 / 11$ & 55919.57 & $\ldots$ & $\ldots$ & $\ldots$ & $21.42(0.19)$ & $\ldots$ & 1 \\
\hline $21 / 01 / 12$ & 55948.41 & $\ldots$ & $\ldots$ & $\ldots$ & $20.12(0.47)$ & $\ldots$ & 3 \\
\hline $30 / 01 / 12$ & 55957.47 & $\ldots$ & $\ldots$ & $\ldots$ & $19.87(0.22)$ & $\ldots$ & 3 \\
\hline $17 / 03 / 12$ & 56003.51 & $\ldots$ & $\ldots$ & $\ldots$ & $19.40(0.07)$ & $\ldots$ & 3 \\
\hline $26 / 03 / 12$ & 56013.36 & $\ldots$ & $\ldots$ & $\ldots$ & $19.13(0.08)$ & $\ldots$ & 3 \\
\hline $28 / 03 / 12$ & 56015.45 & $\ldots$ & $\ldots$ & $\ldots$ & $19.23(0.08)$ & $\ldots$ & 3 \\
\hline $17 / 07 / 12$ & 56126.34 & $\ldots$ & $\ldots$ & $\ldots$ & $>20.90$ & $\ldots$ & 3 \\
\hline $26 / 07 / 12$ & 56135.35 & $\ldots$ & $\ldots$ & $\ldots$ & $>20.70$ & $\ldots$ & 3 \\
\hline $26 / 10 / 12$ & 56226.26 & $\ldots$ & $>20.12$ & $>20.16$ & $>19.82$ & $>19.43$ & 5 \\
\hline $06 / 11 / 12$ & 56237.63 & $\ldots$ & $\ldots$ & $\ldots$ & $>21.35$ & $\ldots$ & 3 \\
\hline $08 / 11 / 12$ & 56239.66 & $\ldots$ & $\ldots$ & $\ldots$ & $21.80(0.24)$ & $\ldots$ & 3 \\
\hline $05 / 12 / 12$ & 56266.73 & $\ldots$ & $\ldots$ & $\ldots$ & $>20.97$ & $\ldots$ & 3 \\
\hline $19 / 12 / 12$ & 56280.59 & $\ldots$ & $20.33(0.10)$ & $19.96(0.09)$ & $19.45(0.07)$ & $19.05(0.11)$ & 3 \\
\hline
\end{tabular}

Notes.

$12.2 \mathrm{~m}$ Calar Alto Telescope + CAFOS, Calar Alto, Almeria (Spain).

$22.56 \mathrm{~m}$ NOT + ALFOSC, La Palma, Canary Islands (Spain).

$31.82 \mathrm{~m}$ Copernico Telescope + AFOSC, Mt. Ekar, Asiago (Italy).

${ }^{4} 4.2 \mathrm{~m}$ WHT + ACAM, La Palma, Canary Islands (Spain).

5 2.0 m Liverpool Telescope + RATCam, La Palma, Canary Islands (Spain).

Table 5

Optical, Swift/UVOT, and NIR Magnitudes of the Reference Stars in the Field of SN 2009ip

\begin{tabular}{|c|c|c|c|c|c|c|c|c|c|c|c|}
\hline Filter & Star 1 & Star 2 & Star 3 & Star 4 & Star 5 & Star 6 & Star 7 & Star 8 & Star 9 & Star 10 & Star 11 \\
\hline$U$ & $15.70(0.01)$ & $16.14(0.04)$ & & & $21.06(0.07)$ & $20.44(0.05)$ & & & & $20.33(0.02)$ & $19.37(0.02)$ \\
\hline$B$ & $15.76(0.02)$ & $16.20(0.01)$ & $21.13(0.02)$ & $19.10(0.05)$ & $20.14(0.04)$ & $20.01(0.02)$ & $20.76(0.03)$ & $20.10(0.01)$ & $20.95(0.02)$ & $19.38(0.02)$ & $20.00(0.03)$ \\
\hline$V$ & $15.20(0.01)$ & $15.62(0.03)$ & $19.56(0.02)$ & $17.68(0.01)$ & $19.08(0.02)$ & $19.17(0.01)$ & $19.47(0.01)$ & $18.96(0.01)$ & $19.70(0.03)$ & $18.37(0.02)$ & $19.66(0.02)$ \\
\hline$R$ & $14.87(0.01)$ & $15.26(0.01)$ & $18.67(0.01)$ & $16.81(0.01)$ & $18.39(0.01)$ & $18.64(0.01)$ & $18.58(0.02)$ & $18.19(0.01)$ & $18.73(0.02)$ & $17.74(0.01)$ & $19.23(0.01)$ \\
\hline$I$ & $14.54(0.01)$ & $14.94(0.01)$ & $17.76(0.02)$ & $15.99(0.02)$ & $17.80(0.02)$ & $18.18(0.02)$ & $17.59(0.01)$ & $17.47(0.01)$ & $17.68(0.01)$ & $17.15(0.02)$ & $18.96(0.01)$ \\
\hline$U V w 2$ & $18.34(0.15)$ & $18.81(0.13)$ & $\ldots$ & $\ldots$ & $\ldots$ & $\ldots$ & & .. & $\ldots$ & & $20.31(0.16)$ \\
\hline$U V m 2$ & $18.20(0.18)$ & $18.61(0.15)$ & $\ldots$ & & & & & & & & $19.67(0.16)$ \\
\hline$U V w 1$ & $16.92(0.08)$ & $17.39(0.07)$ & $\ldots$ & $\ldots$ & $\ldots$ & $\ldots$ & $\ldots$ & $\ldots$ & $\ldots$ & $\ldots$ & $19.45(0.17)$ \\
\hline$u$ & $15.64(0.05)$ & $16.01(0.05)$ & $\ldots$ & $\ldots$ & $\ldots$ & $\ldots$ & $\ldots$ & $\ldots$ & $\ldots$ & $20.25(0.20)$ & $19.05(0.21)$ \\
\hline$b$ & $15.70(0.07)$ & $16.19(0.05)$ & $\ldots$ & $\ldots$ & $20.10(0.27)$ & $19.84(0.33)$ & $20.60(0.34)$ & $\ldots$ & $20.70(0.19)$ & $19.33(0.20)$ & $20.17(0.22)$ \\
\hline$v$ & $15.28(0.07)$ & $15.65(0.09)$ & $\ldots$ & $\ldots$ & $19.23(0.22)$ & $19.09(0.21)$ & $19.37(0.21)$ & $\ldots$ & $19.73(0.16)$ & $18.50(0.22)$ & $19.98(0.28)$ \\
\hline$J$ & $14.12(0.03)$ & $14.43(0.03)$ & $\ldots$ & $15.05(0.05)$ & $\ldots$ & $\ldots$ & $16.61(0.15)$ & $16.75(0.10)$ & $16.61(0.15)$ & $16.58(0.15)$ & \\
\hline$H$ & $13.84(0.04)$ & $14.02(0.05)$ & $\ldots$ & $14.35(0.05)$ & $\ldots$ & $\ldots$ & $15.85(0.18)$ & $15.90(0.19)$ & $15.83(0.18)$ & $15.80(0.17)$ & $\ldots$ \\
\hline Ks & $13.71(0.06)$ & $14.13(0.07)$ & $\ldots$ & $14.18(0.07)$ & $\ldots$ & $\ldots$ & $\ldots$ & $15.56(0.24)$ & $15.73(0.26)$ & $15.29(0.17)$ & $\ldots$ \\
\hline
\end{tabular}

Note. The NIR magnitudes are taken from the 2MASS catalog. 


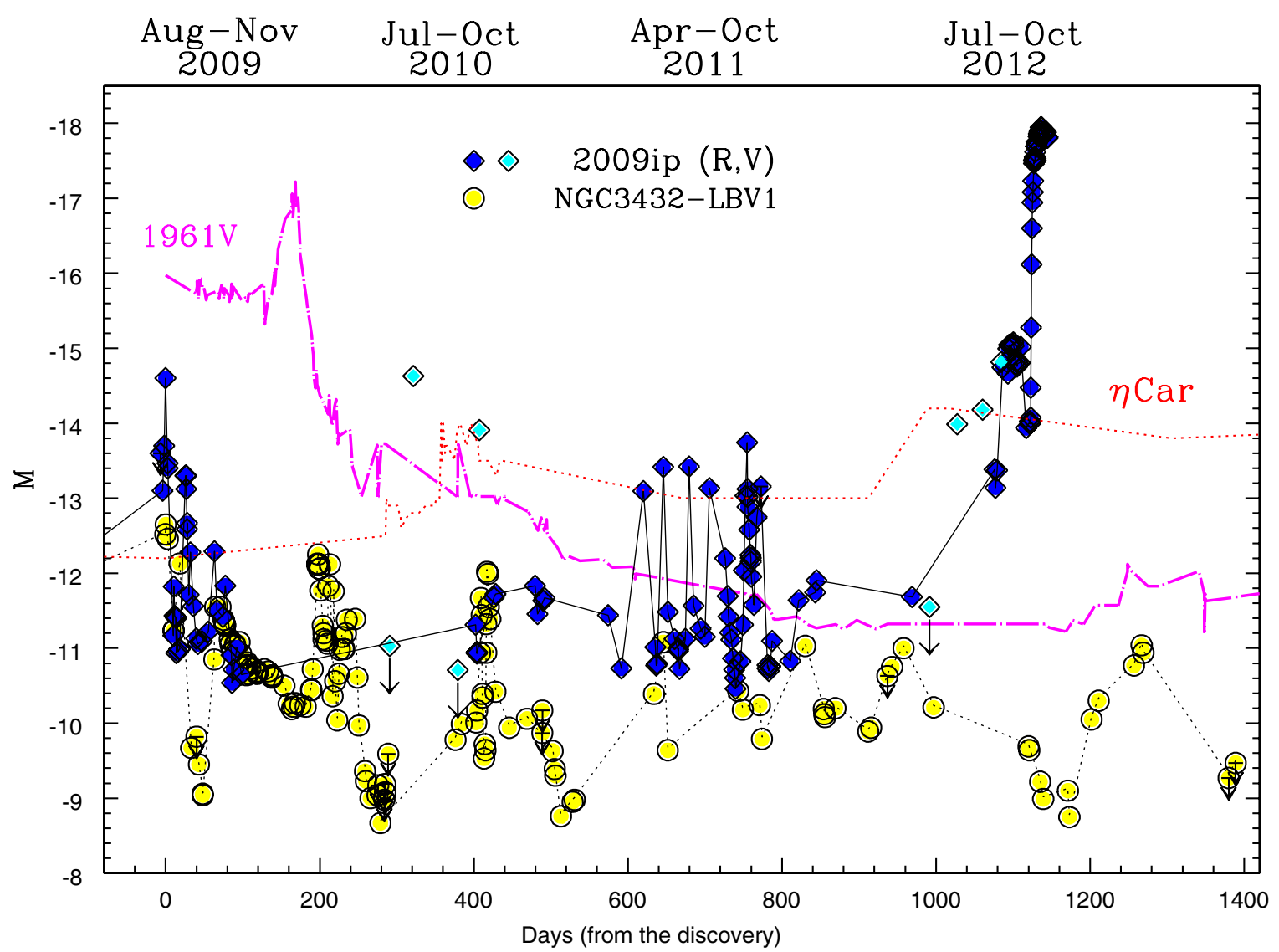

Figure 2. $R$-band absolute light curve of SN 2009ip (blue diamonds) compared with those of the impostor NGC 3432-LBV1 (yellow circles), the debated SN/impostor $1961 \mathrm{~V}$ (photographic plate magnitudes, magenta dot-dashed line), and the historical visual light curve of $\eta$ Carinae during the period $1842-1845$ (revised by Smith \& Frew 2011, red dotted line). The cyan diamonds represent CRTS V-band measurements (see also Drake et al. 2010, 2012). The data showing NGC 3432-LBV1 during the period 2008-2012 are from Pastorello et al. (2010), plus additional recent unpublished observations (see Table 4). The epoch 0 of the $\eta$ Carinae light curve is year 1842.213 (UT). The erratic photometric variability is a common property of major eruptions of LBVs.

(A color version of this figure is available in the online journal.)

2010 September 29, Drake et al. 2010, shown as cyan diamonds in Figure 2). Older records (before 2009 August) from the CRTS archive $^{32}$ and from Smith et al. (2010) have never registered the transient at a magnitude brighter than about $\sim 20.4$. These new data are more comprehensive, and reveal a recent variability history for SN 2009ip which is more complex than one can infer from the schematic light curve representation of Mauerhan et al. (2012a).

During 2012 July-August a new re-brightening was announced by Drake et al. (2012, cyan diamonds in Figure 2). This event was then followed by a strong unprecedented burst (starting around September 23) which is about 30 times more luminous than the previous oscillations. This SN-like rise in luminosity will be extensively discussed later in this paper.

\subsection{Bolometric Light Curve}

A pseudo-bolometric light curve was derived integrating the observed fluxes of SN 2009ip from the ultraviolet to the NIR domains. In practice, for each epoch that had $R$-band observations available and for each band we derived the flux at the effective wavelength. When no observation in a given $X$ filter was available for a specific epoch, the missing $X$-band photometric point was obtained through an interpolation of the available data or, if necessary, by extrapolating the missing

32 http://nesssi.cacr.caltech.edu/catalina/current.html photometry assuming a constant $(R-X)$ color from the first/last available epoch. The fluxes, corrected for the adopted extinction, provide the spectral energy distribution at the given phase, which is then integrated by the trapezoidal rule. The observed flux is finally converted into luminosity adopting the distance value mentioned above. We did not account for flux contribution outside the observed ultraviolet to NIR bands, and therefore this should be more properly quoted as a "quasi-bolometric" light curve. Error estimates include the error in the photometry listed in Tables 1,2, and 3, and the uncertainty in the distance modulus.

Figure 4 shows the bolometric light curve of SN 2009ip from the 2012 August re-brightening announced by Drake et al. (2012) to the current epoch. It appears to show two distinct phases: a broader (and fainter) earlier peak (that we will label as "2012a event" for simplicity) that ends around September 23 and reaches a luminosity of $3 \times 10^{41} \mathrm{erg} \mathrm{s}^{-1}$, and a fast-rising, higher luminosity second peak ("2012b event") with a maximum at about $8 \times 10^{42} \mathrm{erg} \mathrm{s}^{-1}$. Mauerhan et al. (2012a) noted that the maximum luminosity of the 2012a event is consistent with the luminosity of a faint SN IIP (Pastorello et al. 2004), although with a faster evolving light curve. Along with spectral similarities, this led Mauerhan et al. (2012a) to suggest that the 2012a event was the actual core-collapse SN event of the LBV star. We confirm that the bolometric luminosity of the 2012a event is similar to SN $2005 \mathrm{cs}$, as one can note from Figure 4. The subsequent faster rise to the second peak (the $2012 \mathrm{~b}$ event) 

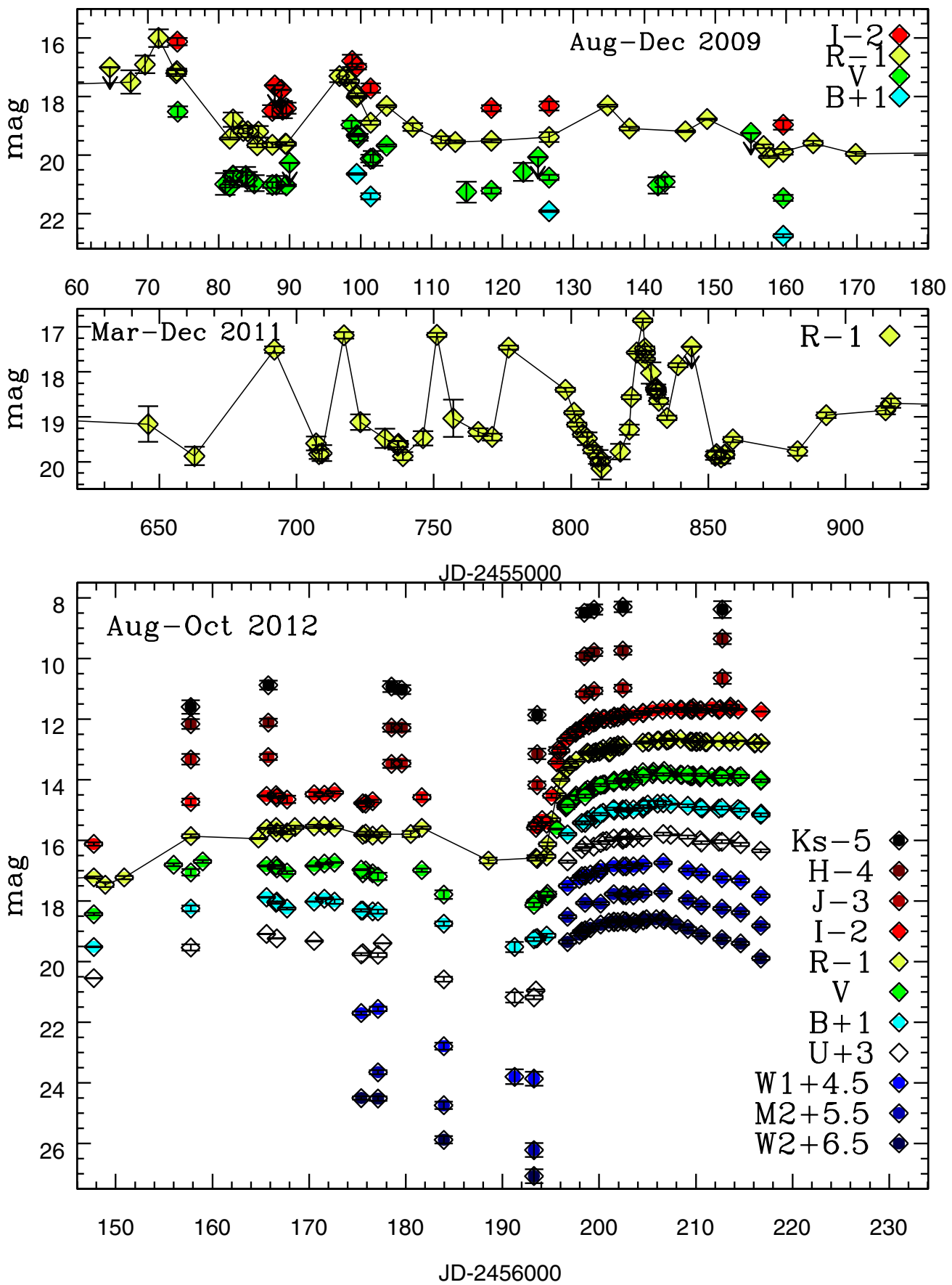

Figure 3. Top: BVRI light curves of the impostor SN 2009ip during the first three months from the first ever detection in 2009 (Maza et al. 2009). Middle: $R$-band light curve of SN 2009ip during the period 2011 March-December, showing erratic magnitude oscillations with $\Delta R \approx 3$ mag. Bottom: ultraviolet/optical/near-infrared apparent light curves of the transient from 2012 August 8, two weeks before the publication of the announcement of a new re-brightening from Drake et al. (2012). Shifts of $\Delta U=+0.27, \Delta B=+0.018$, and $\Delta V=-0.042$ have been applied to the $u, b, v$ Swift/UVOT magnitudes of SN 2009ip to match the $U, B, V$ Johnson photometry. The shifts have been computed after a comparison of the magnitudes of the reference stars in the SN field in the two photometric systems.

(A color version of this figure is available in the online journal.)

presents an even tighter similarity with that of the type IIn/IIL SN 1998S. The 2012b event was proposed by Mauerhan et al. (2012a) to be due to strong SN ejecta-circumstellar medium (CSM) interaction. We measure a two-week-long rise time, reaching a peak apparent magnitude of $B=13.80(R=13.65)$ on 2012 October 6 and then it declines in luminosity, more rapidly in the ultraviolet and the blue optical bands. We will see in Section 3 that the 2012a and 2012b sequence of events may have an alternative explanation.
We also remark that none of the comparison objects in Figure 2 shows the regular, SN-like light curve that characterized SN 2009ip during the $2012 \mathrm{~b}$ event. This late photometric evolution combined with the bright luminous peak $\left(M_{R} \approx-18\right)$ may support the claim that at least during the $2012 \mathrm{~b}$ event SN 2009ip has finally exploded as a real SN. We note that the color of SN 2009ip at the light curve peak (on 2012 October 6) is $U-V \approx-1 \mathrm{mag}$, significantly bluer than that of the 2012a event at maximum $(U-V \approx-0.5 \mathrm{mag})$. At the pre-burst minimum of 


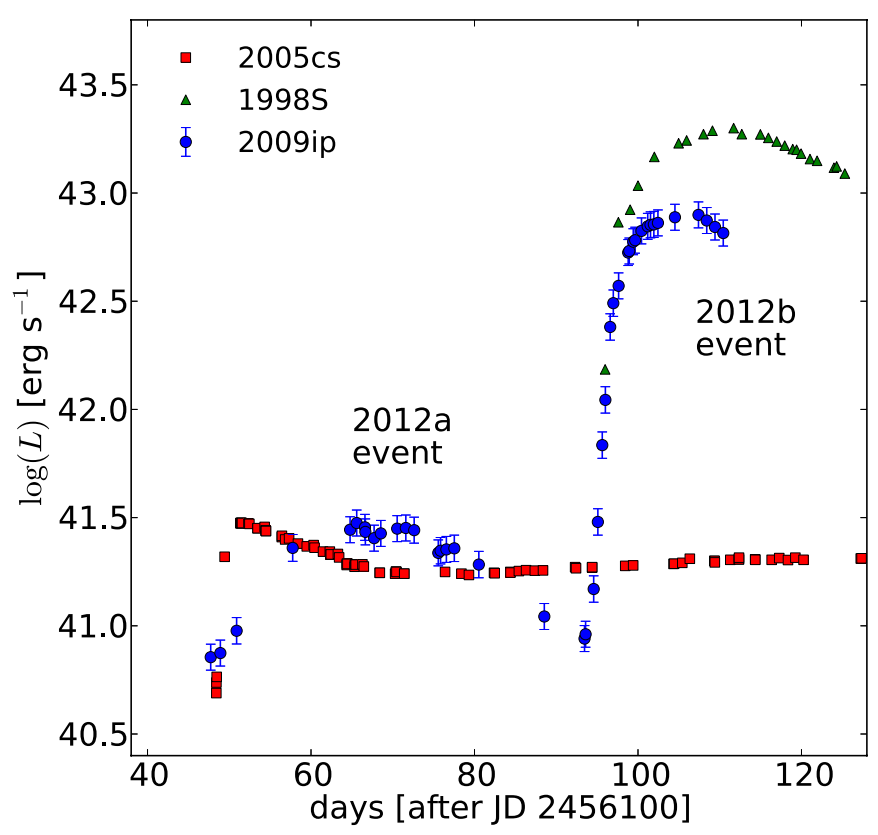

Figure 4. Bolometric light curve of SN 2009ip from 2012 August to October (showing both the 2012a and 2012b events), compared with the bolometric light curves of the faint type IIP SN 2005cs (Brown et al. 2007; Pastorello et al. 2006, 2009) and the type IIn/IIL SN 1998S (Liu et al. 2000; Fassia et al. 2000; Gerardy et al. 2002; Pozzo et al. 2004). The light curves of SNe 2005cs and $1998 \mathrm{~S}$ are shown in an arbitrary temporal scale to match well, respectively, the 2012a and 2012b eruptive events of SN 2009ip.

(A color version of this figure is available in the online journal.)

September 23 , the $U-V$ color was instead significantly redder, i.e., $\approx 0$ mag.

\subsection{Spectroscopy}

Optical and near-infrared spectra of SN 2009ip (Figures 5-9) were collected using the $8.2 \mathrm{~m}$ VLT UT1 (+ FORS2) and UT2 (+XShooter) at the Cerro Paranal Observatory (ESO Chile), the $3.58 \mathrm{~m}$ ESO-NTT (+ EFOSC2 and SOFI) at the La Silla Observatory (ESO Chile), the $8.2 \mathrm{~m}$ Gemini North Telescope (with GMOS) in Cerro Pachón (Chile), the $3.58 \mathrm{~m}$ TNG (equipped with LRS), the $4.2 \mathrm{~m}$ William Herschel Telescope (WHT, with ISIS), and the $2.56 \mathrm{~m}$ NOT (+ ALFOSC) located in La Palma (Canary Islands, Spain). Basic information on the spectra collected during the observational campaign of SN 2009ip is reported in Table 6.

The spectroscopy data reduction steps were performed using IRAF tasks. The pre-reduction process (i.e., overscan and bias corrections, flat-fielding, and trimming) is the same as described for the photometry images. In addition, for the IR spectra, the contribution of the night sky emission was removed by subtracting from each other two consecutive exposures taken with the source in different positions along the slit. The optimal extraction of one-dimensional spectra allowed us to remove the flux contamination of the night sky (for the optical spectra) and other background sources. The spectra were then wavelength calibrated using reference spectra of arc lamps, and calibrated in flux using sensitivity curves obtained through spectra of spectrophotometric standards. The consistency of the spectroscopic flux calibration was finally checked using the available SN photometry and, when discrepant, the spectral fluxes were rescaled. Telluric standards were used to correct the NIR spectra for the effects of the broad atmospheric absorption bands.
The spectra relative to the 2009 outburst reported in Figure 5 are all dominated by prominent Balmer lines with a complex profile. The weak absorption features indicate that the bulk of the ejected material is moving with a velocity of $2900 \pm 700 \mathrm{~km} \mathrm{~s}^{-1}$, but the blue edge of the isolated $\mathrm{H} \beta$ absorption suggests the presence of fast-moving material which is expanding at a velocity of about $5000-6000 \mathrm{~km} \mathrm{~s}^{-1}$. The $\mathrm{H} \alpha$ emission component in 2009 September has a Lorentzian profile with an FWHM velocity of about $700-800 \mathrm{~km} \mathrm{~s}^{-1}$, which increases to about $1100-1200 \mathrm{~km} \mathrm{~s}^{-1}$ during the period 2009 October-November (when the object was receding to a more quiescent stage).

After a further outburst (2010 September) reported by Drake et al. (2010), a spectrum obtained on 2010 October 6 shows SN 2009ip at a similar stage as the 2009 November 24 spectrum, i.e., with the star again quiescent. The FWHM velocity of the Lorentzian $\mathrm{H} \alpha$ component in this phase is still around $1300 \mathrm{~km} \mathrm{~s}^{-1}$. The 2011 September 2 VLT spectrum reported at the bottom of Figure 5 shows SN 2009ip to be back to a dormant stage, and the FWHM velocity of the Lorentzian $\mathrm{H} \alpha$ component is about $940 \mathrm{~km} \mathrm{~s}^{-1}$.

Figure 6 shows the spectra of the transient during the period 2012 August-October, compared with a VLT spectrum obtained on 2011 September 24 (green line), during another outburst episode. In the 2011 September 24 spectrum, the FWHM velocity of $\mathrm{H} \alpha$, which still has a Lorentzian profile, has slightly decreased to around $790 \mathrm{~km} \mathrm{~s}^{-1}$, and other Balmer lines clearly show very broad absorption components, with a blue edge that indicates that there is material moving with a velocity as high as $12,500 \mathrm{~km} \mathrm{~s}^{-1}$ already at this epoch (see also Figure 7). This is the highest velocity outflow that has been detected in an LBV-like eruption of any sort and indicates that high velocities are observed without core collapse or the catastrophic destruction of the star. This has important consequences for the interpretation of high-velocity ejecta as evidence for the corecollapse mechanism in the $2012 \mathrm{a}$ event. ${ }^{33}$

We subsequently obtained an NTT spectrum on 2012 August 8 (JD $=2456148.91$, i.e., 10 days before the new outburst - the 2012a event-was announced by Drake et al. 2012). The broad absorption features were also present at this epoch, and indeed were stronger than in the 2011 September 24 spectrum (Figure 6). The minimum of the broad absorption components of the Balmer lines has a core at $8600 \pm 400 \mathrm{~km} \mathrm{~s}^{-1}$, with a blue wing extending up to $14,000 \mathrm{~km} \mathrm{~s}^{-1}$, while the Lorentzian emission survives at an FWHM velocity of about $1380 \mathrm{~km} \mathrm{~s}^{-1}$. The presence of these components was observed in 2012 September 15 and 16 spectra by Smith \& Mauerhan (2012a), and this was the critical measurement that led the authors to propose that the LBV had exploded as a core-collapse SN, i.e., the 2012a event was due to stellar core collapse and an explosion with fairly low kinetic energy like SN 2005cs.

Our spectra collected between 2012 August 18 and September 5 show little evolution: the $\mathrm{H}$ features show prominent P-Cygni profiles, with deep minima at 8000-9000 $\mathrm{km} \mathrm{s}^{-1}$ and edges possibly extending to $14,000-15,000 \mathrm{~km} \mathrm{~s}^{-1}$. The $\mathrm{H} \alpha$ narrow emission component still has an FWHM velocity of $800 \pm 100 \mathrm{~km} \mathrm{~s}^{-1}$, while the highest resolution spectra allow us

\footnotetext{
33 We are confident that the broad-line absorptions observed in the 2011 September XShooter spectrum are intrinsic to the object and not artifacts due to the instrumental effects. In fact, the major problem that could affect the line shapes (in particular the absorptions) could be the blaze function not being properly corrected at the edges of the spectral orders. However, the resulting patterns would affect a wider wavelength range $(\sim 1000 \AA)$ and would have a smoother effect rather than mimic a single line absorption profile (see, e.g., the 2010 March 23 spectrum of SN 2010bh in Bufano et al. 2012).
} 


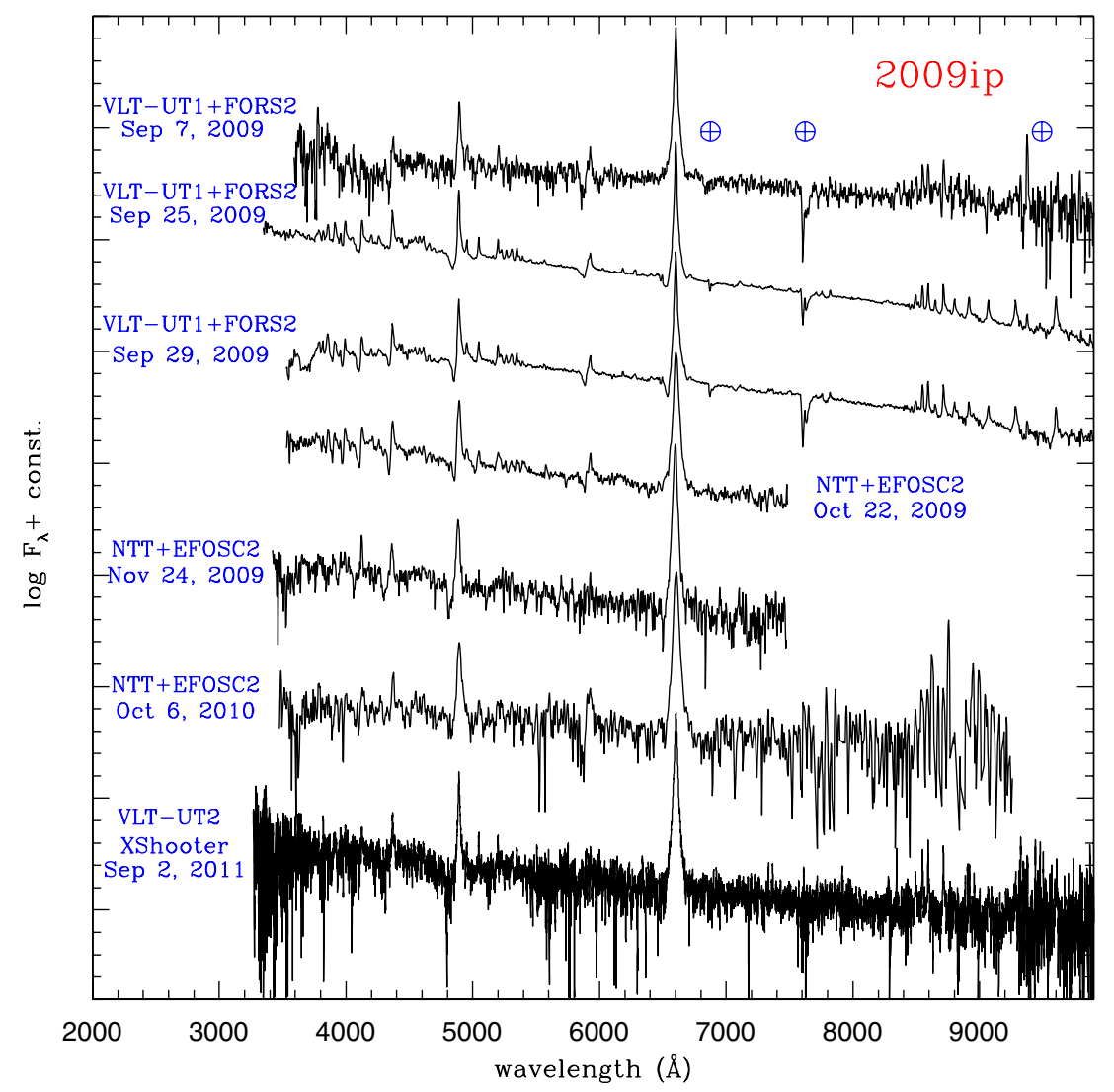

Figure 5. Sequence of spectra of the LBV in NGC 7259, obtained from 2009 September to 2011 September. All spectra are in the host galaxy wavelength frame. The symbols " $\oplus$ " mark the positions of the strongest telluric absorption bands.

(A color version of this figure is available in the online journal.)

Table 6

Log of Observed Spectra of SN 2009ip

\begin{tabular}{lcccc}
\hline \hline Date (dd/mm/yy) & JD-2,400,000 & Instrumental Configuration & Range $(\AA)$ & Resolution $(\AA ̊)$ \\
\hline $07 / 09 / 09$ & 55081.57 & VLT(UT1)+FORS2+300V+300I & $3500-10350$ & $10 ; 9$ \\
$25 / 09 / 09$ & 55099.58 & VLT(UT1)+FORS2+300V+300I & $3250-10000$ & $10 ; 9$ \\
$29 / 09 / 09$ & 55103.66 & VLT(UT1)+FORS2+300V+300I & $3500-10030$ & $10 ; 9$ \\
$22 / 10 / 09$ & 55126.7 & NTT+EFOSC2+gm11+gm16 & $3530-9440$ & $14 ; 12$ \\
$24 / 11 / 09$ & 55159.58 & NTT+EFOSC2+gm11 & $3350-7470$ & 14 \\
$06 / 10 / 10$ & 55475.60 & NTT+EFOSC2+gm11+gm16 & $3360-9540$ & $21 ; 20$ \\
$02 / 09 / 11$ & 55807.48 & VLT(UT2)+XShooter & $3030-10400$ & $1.0 ; 0.8$ \\
$24 / 09 / 11$ & 55828.64 & VLT(UT2)+XShooter & $3150-22900$ & $1.0 ; 0.8 ; 2.8$ \\
$08 / 08 / 12$ & 56148.93 & NTT+EFOSC2+gm11 & $3360-7470$ & 14 \\
$18 / 08 / 12$ & 56157.76 & VLT(UT2)+XShooter & $3100-24790$ & $1.0 ; 0.8 ; 2.8$ \\
$25 / 08 / 12$ & 56164.77 & NTT+EFOSC2+gm11 & $3390-7450$ & 14 \\
$26 / 08 / 12$ & 56165.58 & NTT+EFOSC2+gm11 & $3360-7450$ & 14 \\
$26 / 08 / 12$ & 56165.78 & NTT+SOFI+GB & $9370-16440$ & 27 \\
$27 / 08 / 12$ & 56166.64 & NTT+EFOSC2+gm13 & $3650-9250$ & 18 \\
$29 / 08 / 12$ & 56168.50 & TNG+Dolores+LRB+LRR & $3170-9800$ & $10.5 ; 9.5$ \\
$30 / 08 / 12$ & 56169.54 & TNG+Dolores+LRB & $3280-8080$ & 10.5 \\
$31 / 08 / 12$ & 56170.50 & TNG+Dolores+LRB+LRR & $3280-9300$ & $14 ; 13$ \\
$05 / 09 / 12$ & 56175.55 & NOT+ALFOSC+gm4 & $3350-9070$ & 18 \\
$10 / 09 / 12$ & 56180.56 & NTT+EFOSC2+gm11+gm16 & $3360-10040$ & $22 ; 20$ \\
$18 / 09 / 12$ & 56188.55 & NTT+EFOSC2+gm11 & $3360-7450$ & 14 \\
$21 / 09 / 12$ & 56192.43 & WHT+ISIS+R300B+R158R & $3200-9250$ & $4.3 ; 7.2$ \\
$22 / 09 / 12$ & 56192.52 & TNG+Dolores+LRB & $3320-8080$ & 14 \\
$23 / 09 / 12$ & 56193.50 & WHT+ISIS+R300B+R158R & $3180-9490$ & $8.6 ; 14$ \\
$23 / 09 / 12$ & 56193.53 & NTT+SOFI+GB+GR & $9370-25200$ & $27 ; 30$ \\
$28 / 09 / 12$ & 56198.80 & GN+GMOS+R400+B600 & $3400-9130$ & $2.7 ; 3.7$ \\
$04 / 10 / 12$ & 56205.40 & TNG+Dolores+LRB & $3320-8090$ & 10 \\
\hline
\end{tabular}




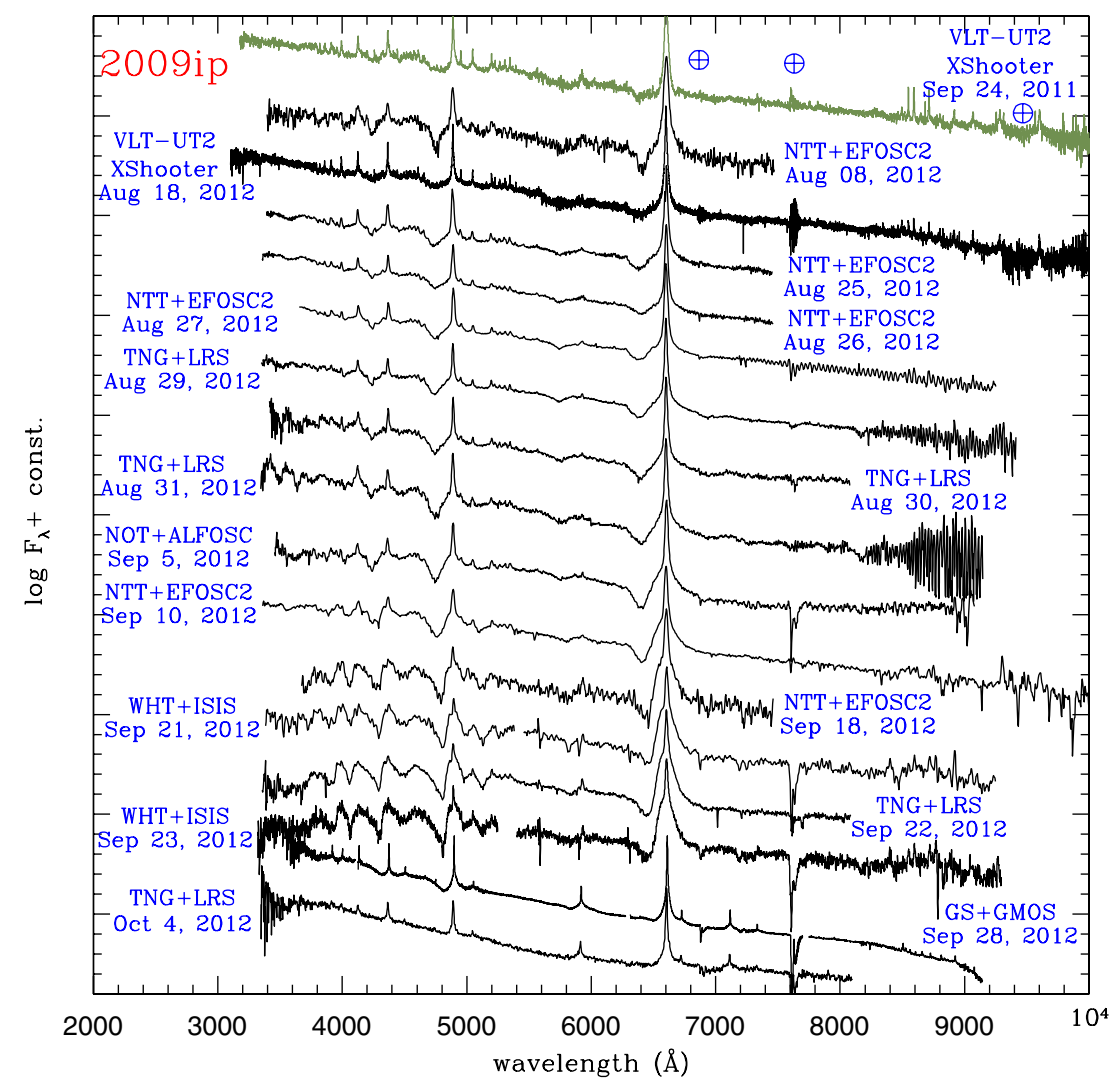

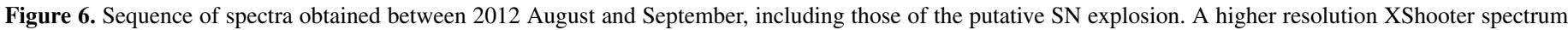

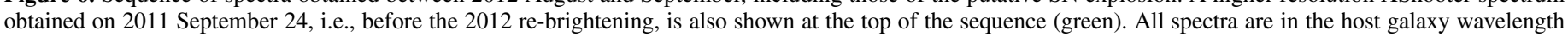
frame. The symbols " $\oplus$ " mark the positions of the strongest telluric absorption bands.

(A color version of this figure is available in the online journal.)

to measure the FWHM velocity from the clearly detected narrow Fe II emissions (multiplet 42) to be about $240 \pm 20 \mathrm{~km} \mathrm{~s}^{-1}$.

As highlighted by Mauerhan et al. (2012a), the spectra from September 10 to 23 (2012a event) do closely resemble those of Type II SNe (the similarity with early spectra of the under-luminous Type IIP SN 2005cs shown in their Figure 4 is remarkable). Both $\mathrm{H}$ and Fe II lines now show broad P-Cygni profiles with a prominent broad emission component. However, we now present spectra of the 2012a event covering a period from 2012 August 8 to 2012 September 23 (47 days), and we do not observe the typical evolution of a Type II SN over this period. In particular, 15-20 days after explosion, Type II-P $\mathrm{SNe}$ develop the strong, broad near-infrared Ca II triplet feature (Pastorello et al. 2006), but we do not observe this for the 2012a event. The cores of the absorptions of the Balmer features indicate an expansion velocity of the ejected material of $\sim 5000-6000 \mathrm{~km} \mathrm{~s}^{-1}\left(4200 \pm 500 \mathrm{~km} \mathrm{~s}^{-1}\right.$ from the Fe II lines), but the blue edge of the wings still reach to much higher velocities (about 13,800 $\mathrm{km} \mathrm{s}^{-1}$ ). Figure 7 (top) shows a comparison of SN 2009ip at three representative epochs (2011 September 24, 2012 August 8, and 2012 September 28) with a spectrum of NGC 3432-LBV1 in outburst (2009 April 24). The high-velocity P-Cygni absorption (in the Balmer lines) is certainly stronger in the 2012a event than we observed in 2011 and in NGC 3432-LBV1 in outburst, but we illustrate here that the detection of high-velocity gas is not only restricted to corecollapse SNe. Similar high-velocity edges are clearly detected in SN 2009ip in $2011\left(13,800 \mathrm{~km} \mathrm{~s}^{-1}\right)$ and in NGC 3432-LBV1
( $~ 9000 \mathrm{~km} \mathrm{~s}^{-1}$; Pastorello et al. 2010). A blowup of the $\mathrm{H} \alpha$ and $\mathrm{H} \beta$ regions of a few selected spectra of SN 2009ip is shown in Figure 8, supporting our claim that broad absorption features - though fainter-were detected even before 2012 August. We will discuss the implications of this in Section 3.

These broad absorptions disappear at the time of the $2012 \mathrm{~b}$ event, in September 28 and October 4 spectra (Figures 6 and 7, top), when the luminosity of SN 2009ip reaches the unprecedented maximum. At these times, the spectra are very similar to those of many Type IIn SNe (e.g., SN 1999el; Di Carlo et al. 2002), with the $\mathrm{H}$ lines presenting a narrow emission component with an FWHM velocity of about $290 \mathrm{~km} \mathrm{~s}^{-1}$ and very broad wings $\left(\sim 3600 \mathrm{~km} \mathrm{~s}^{-1}\right)$. Similar velocities are measured in the He I lines, which are now more prominent than in past spectra, while the Fe II lines are no longer visible.

The spectrum of SN 2009ip obtained on 2009 September 25 with VLT-UT1 equipped with FORS2 has a very high signalto-noise ratio. This gives us the opportunity to identify the most important lines in the spectrum of SN 2009ip (Figure 7, bottom). The spectrum is dominated by strong Balmer and Paschen lines of $\mathrm{H}$, showing weak and narrow $\left(2850 \pm 490 \mathrm{~km} \mathrm{~s}^{-1}\right) \mathrm{P}$-Cygni profiles. Weak He I lines (being the $5876 \AA$ feature blended with Na I D 5890-5896 $\AA$ ) and a number of Fe II multiplet lines are also detected. We note that in the 2012 September 28 spectrum of SN 2009ip (during the 2012b outburst, Figure 7), the spectral properties are quite similar to those observed in the aforementioned VLT spectrum, although the detection of Fe II lines is not obvious. Most of these lines are also 


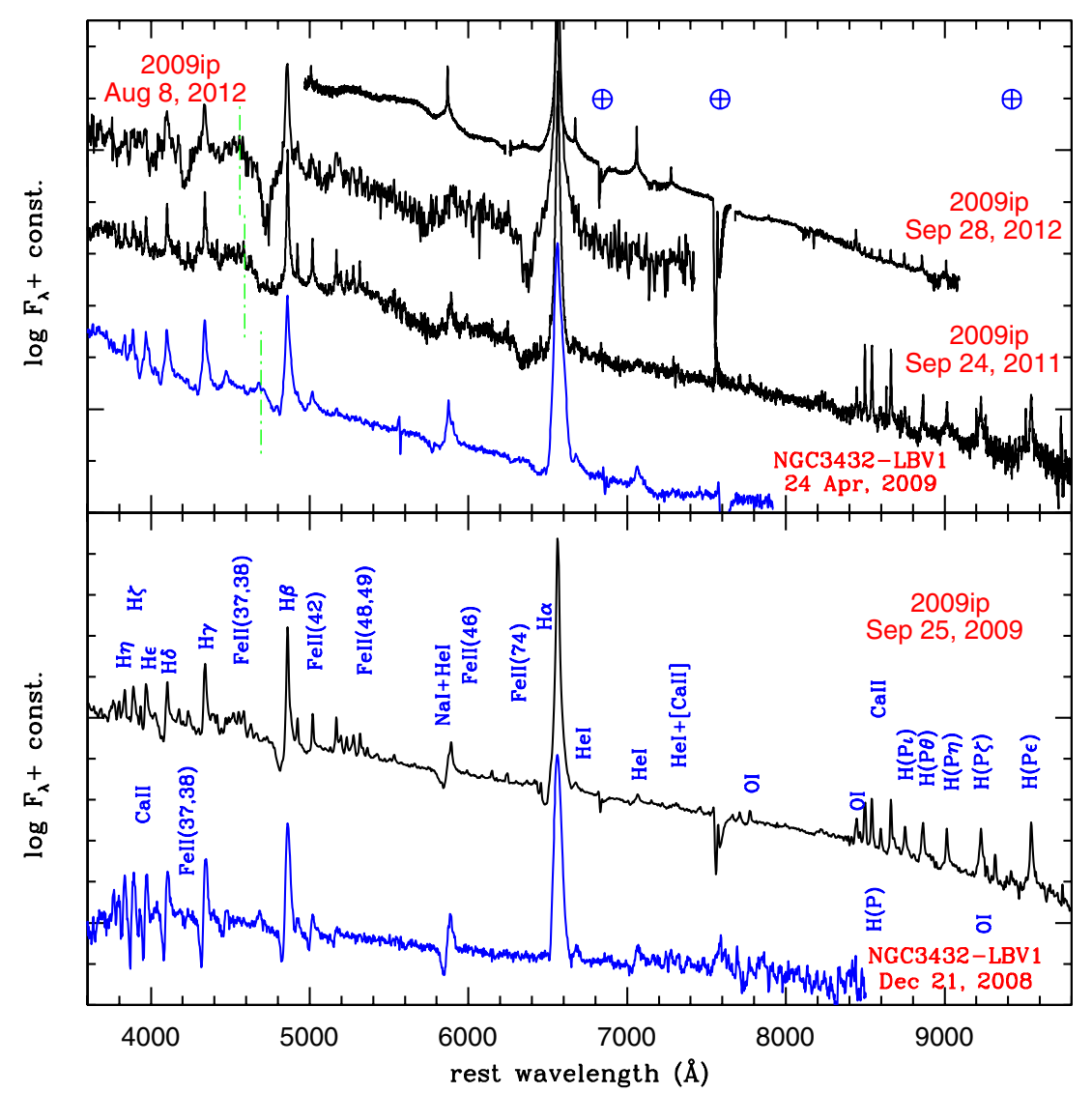

Figure 7. Top: comparison of spectra of SN 2009ip at three representative epochs (2011 September 24, and during the 2012a and 2012b events) with a spectrum in the outburst of NGC 3432-LBV1. The vertical dashed green lines mark the position of the highest velocity edges of the $\mathrm{H} \beta$ components in the two objects. Bottom: line identification in the optical spectrum of SN 2009ip obtained on 2009 September 25 (VLT+FORS2), and comparison with a spectrum of NGC $3432-\mathrm{LBV} 1$ in outburst. The symbols " $\oplus$ " mark the positions of the strongest telluric absorption bands in the spectra of SN 2009ip.

(A color version of this figure is available in the online journal.)

visible in the spectrum of the impostor NGC 3432-LBV1 shown as a comparison, with quite similar velocities of the narrow components $\left(\leqslant 650 \mathrm{~km} \mathrm{~s}^{-1}\right)$. Narrow O I and Ca II lines are relatively prominent in SN 2009ip, while they were not unambiguously detected in NGC 3432-LBV1 (although this might be due to the lower signal-to-noise spectrum).

A sequence of near-infrared spectra of SN 2009ip is shown in Figure 9. The continuum is always quite blue in these spectra. The strongest lines are detected as broad features with P-Cygni profiles, and narrower emissions superimposed to the broad components. The broad P-Cygni components become more evident with time and in the 2012 September 23 spectrum (at the time of the onset of the 2012b eruption) they dominate over the narrow lines. We identify $\mathrm{Br} \gamma$ at $2165 \mathrm{~nm}, \mathrm{~Pa} \alpha$ (which is barely visible in the middle of the telluric absorption around $1875 \mathrm{~nm}), \mathrm{Pa} \beta$ at $1282 \mathrm{~nm}$, and $\mathrm{Pa} \gamma$ at $1094 \mathrm{~nm}$, blended with He I $1083 \mathrm{~nm}$. The 2012 September 23 spectrum, in particular, shows a broad $\mathrm{Pa} \beta$ with an FWHM velocity of about $6200 \mathrm{~km} \mathrm{~s}^{-1}$ and a prominent blueshifted absorption of Pa $\gamma+\mathrm{He}$ I $1083 \mathrm{~nm}$ with an expansion velocity of about $10,000 \mathrm{~km} \mathrm{~s}^{-1}$, as obtained from the position of the broad absorption minimum. The narrow He I $1083 \mathrm{~nm}$ line, which was marginally detectable in previous spectra, is now clearly visible, and is well separated from Pa $\gamma$. The narrow Paschen lines have Lorentzian profiles with an FWHM velocity of about $400 \mathrm{~km} \mathrm{~s}^{-1}$, while the narrow He I $1083 \mathrm{~nm}$ appears to be slightly broader $\left(\sim 800-1000 \mathrm{~km} \mathrm{~s}^{-1}\right)$ and with a roughly Gaussian profile.

\section{REAL SUPERNOVA OR SUPERNOVA IMPOSTOR?}

SN 2009ip is a remarkable object for a number of reasons: (1) it experienced a series of energetic outbursts since 2009, when the transient reached absolute peak magnitudes between -14 and -15 ; (2) the spectral features reveal the presence of ejected material at very high velocities (several $\times 10^{3} \mathrm{~km} \mathrm{~s}^{-1}$ ); (3) the progenitor star was observed to be extremely luminous in quiescence $\left(M_{V}=-10.0 \pm 0.3\right)$ and was proposed to be a massive LBV ( $>60 M_{\odot}$; Foley et al. 2011; Smith et al. 2010); (4) finally, in 2012 September the star displayed a further, exceptionally luminous outburst (the $2012 \mathrm{~b}$ event, with $M_{V} \approx-18$; Mauerhan et al. 2012a and references therein), suggesting that the LBV may have experienced a core-collapse SN explosion. The luminosity during that event and its similarity to SNe IIn spectra are possibly the strongest indicators that a core-collapse SN has occurred, more so than the broad lines of the spectra during the 2012a pre-cursor event.

The complex, erratic 2009-2012 light curve of SN 2009ip (Section 2.1) indicates that the LBV entered a very active variability phase resembling those of the unusual NGC 3432-LBV1 or $\eta$ Carinae during the Giant Eruption of the 19th century. In the case of NGC 3432-LBV1, multiple eruptions on short 


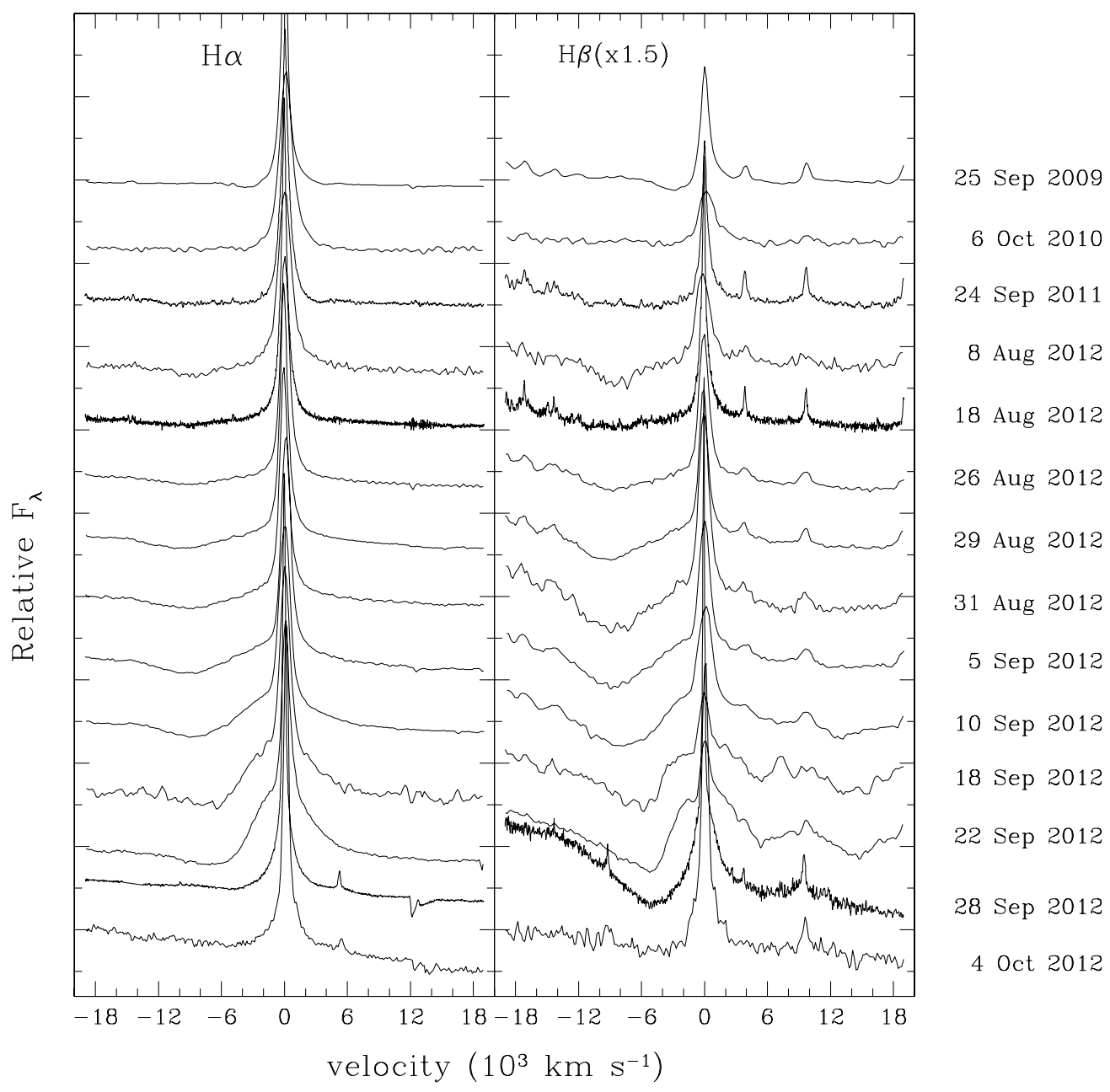

Figure 8. Blowup of the regions of $\mathrm{H} \alpha$ (left) and $\mathrm{H} \beta$ (right) for a selected sub-sample of SN 2009ip spectra. The velocities in abscissa are in units of $10^{3} \mathrm{~km} \mathrm{~s}^{-1}$.

timescales (about 200-220 days) have been proposed to be the result of violent pulses of a very massive star (possibly via the pulsational pair-instability mechanism) that is approaching the end of its life, presumably with the core collapse. Alternatively, the pulses may be regulated by the passage of a companion star to the periastron (Pastorello et al. 2010). ${ }^{34}$

The presence of very fast material $\left(\sim 13,000 \mathrm{~km} \mathrm{~s}^{-1}\right)$ in SN 2009ip already almost one year before the putative $S N$ explosion (i.e., in the 2011 September 24 spectrum) and also in NGC 3432-LBV1 $\left(\sim 9000 \mathrm{~km} \mathrm{~s}^{-1}\right)$ suggests that these LBV related eruptions could quite feasibly be linked with the 2012a event. The highest velocity in the Homunculus Nebula surrounding $\eta$ Carinae reaches $3500-6000 \mathrm{~km} \mathrm{~s}^{-1}$ (Smith 2008). Typical LBV eruptions are discussed in terms of extreme stellar winds driven by the super-Eddington luminosity of the star. However, these winds are expected to have velocities of the order of a few $\times 10^{2} \mathrm{~km} \mathrm{~s}^{-1}$ (e.g., Smith 2008). The detection of this high-velocity gas in some LBV outbursts (including the aforementioned events) suggests that these episodes probably originate in explosions deeper in the star, perhaps in the core. These release an energy that may compete with those of weak SNe (e.g., faint SNe IIP, such as SN 1999br; Pastorello et al. 2004), producing a blast wave that allows the star to expel massive portions of the envelope (Smith 2008). All of this is

\footnotetext{
34 The presence of a companion was proposed to explain the modulated, quasi-periodic light curve of NGC 3432-LBV1 (Pastorello et al. 2010).
}

expected to produce transients that closely mimic the energy and the overall properties of a real SN exploding in a dense CSM (type IIn).

\subsection{No Core-collapse SN During the 2012a Event?}

One of the most remarkable findings inferred from the analysis of the August and early September spectra of SN 2009ip (during the 2012a event) is that the bulk of the ejected material has extremely high expansion velocities $\left(8000-9000 \mathrm{~km} \mathrm{~s}^{-1}\right.$, with edges extending up to $14,000 \mathrm{~km} \mathrm{~s}^{-1}$; see Section 2.3 and Mauerhan et al. 2012a). This, and the striking similarity between the early September spectra of SN 2009ip and those of the weak Type IIP SN 2005cs (Pastorello et al. 2006, 2009), led Mauerhan et al. (2012a) to conclude that SN 2009ip had likely exploded as a faint, ${ }^{56} \mathrm{Ni}$-poor core-collapse $\mathrm{SN}$ during the August rebrightening episode. The fact that we can observe features from the SN ejecta inside an extended and dense CSM is explained with a non-homogeneous, possibly clumpy distribution of the material lost by the LBV in pulsations preceding the explosion. While this is plausible, we would caution that the detection of high-velocity ejecta cannot be regarded as a conclusive proof, because very high velocity material was also observed in NGC 3432-LBV1 (Pastorello et al. 2010, where the broad wing of the $\mathrm{H} \beta$ absorption extended to $9000 \mathrm{~km} \mathrm{~s}^{-1}$ ), during an eruption of a known SN impostor. 


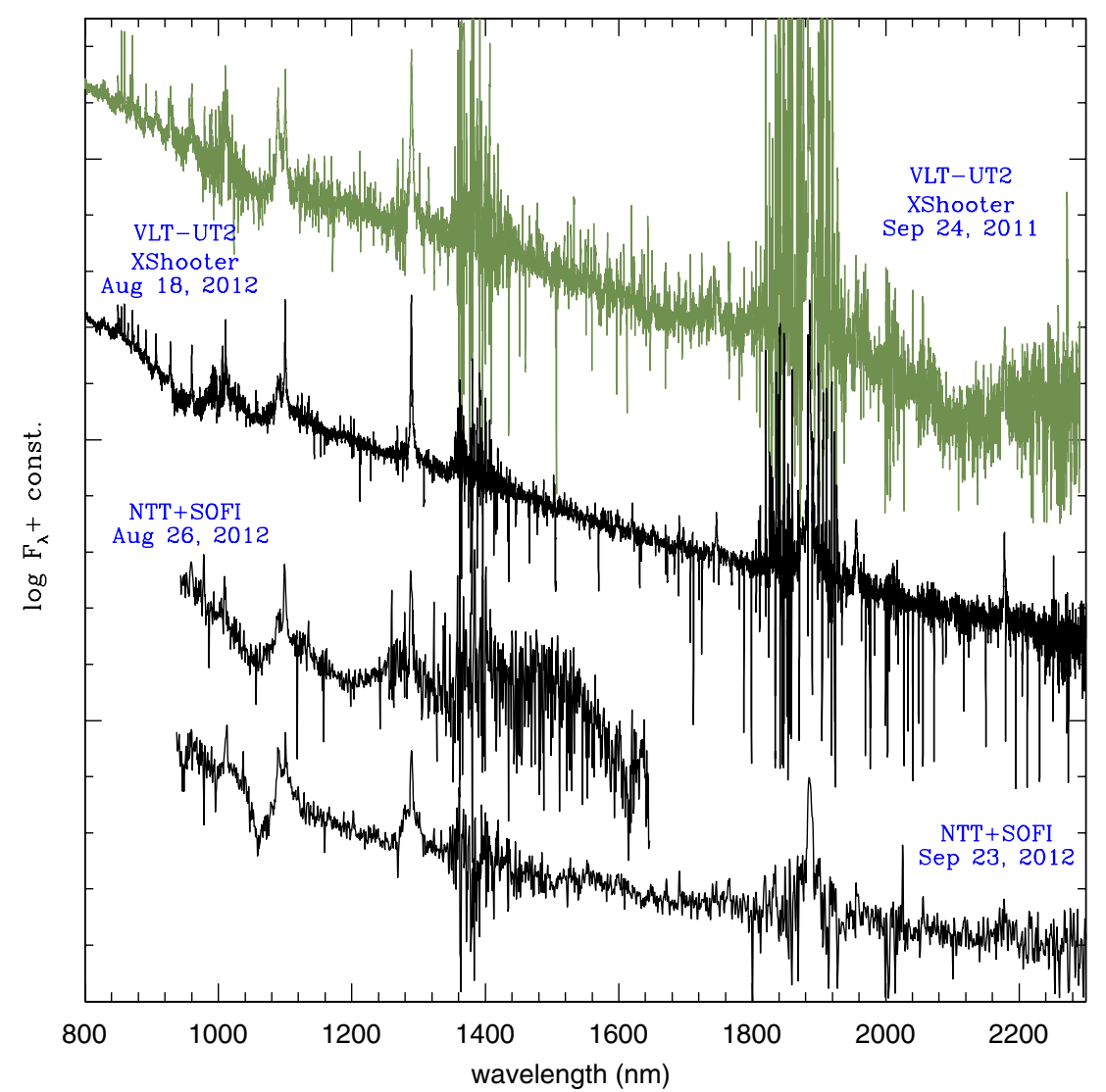

Figure 9. Sequence of near-infrared spectra of SN 2009ip obtained from 2012 August to September. The XShooter spectrum of 2011 September 24 is also shown in green.

(A color version of this figure is available in the online journal.)

The core-collapse SN scenario proposed by Mauerhan et al. (2012a) is questionable, since there is a number of observables that require a rather ad hoc combination of events: (1) the highvelocity absorption wings measured in the spectra obtained after the announcement of the 2012a outburst episode (Drake et al. 2012) are actually similar to those we have seen in the 2011 September 24 and 2012 August 8 spectra, which raises the question whether, and, in case, when the SN explosion occurred; (2) the peak absolute magnitude $\left(M_{R} \sim-15\right)$ and the evolutionary timescales of the 2012a event are consistent with those of previous eruptive episodes (in particular the 2009 event, see Figure 3); finally (3) it is not trivial to explain how an extremely massive LBV $\left(M>60 M_{\odot}\right.$, likely with $M_{\text {ZAMS }} \geqslant 90-100 M_{\odot}$ ) can explode as a weak Type II SN: we may need to invoke subsequent eruptions to explain the events before 2012 July-August, and subsequently a fall-back core-collapse $\mathrm{SN}$ with the formation of a black hole.

In the Mauerhan et al. (2012a) interpretation, the 2012b event is fairly simply explained as a core-collapse SN ejecta-CSM interaction. However it is also plausible that the 2012a event was an eruptive phase, and the 2012b luminosity comes from the actual core collapse, similar to what is assumed to occur in IIn $\mathrm{SNe}$, or from the collision of material ejected in the previous eruption with pre-existing CSM, or even from the illumination of the inner parts of a dense circumstellar disk by faster ejected material (as proposed by Levesque et al. 2012).

An alternative explanation for the nature of the 2012a outburst has been offered by Soker \& Kashi (2013), who noted some similarity between the $2012 a+2012 b$ light curve of SN 2009ip with that of the unusual eruptive variable V838Mon (Tylenda
2005; Tylenda \& Soker 2006), and proposed that the ejection of fast material following the merging of two massive stars might explain the 2012a event, while the subsequent collision of this fast material with pre-existing CSM would produce the $2012 \mathrm{~b}$ event.

\subsection{SN 2009ip, A Pulsational Pair-instability Event}

The detection of high-velocity ejecta $\left(12,500 \mathrm{~km} \mathrm{~s}^{-1}\right)$ on 2011 September 24 indicates that the star has managed to eject material at velocities that we would normally associate with an SN explosion. It is very unlikely that the core collapsed at this point (see Section 3.1), which implies that the high-velocity material has been ejected in the 2012a event without invoking a core-collapse SN explosion. What triggers these ejections is still unclear, but the very high progenitor mass (Smith et al. 2010; Foley et al. 2011) indicates that the events may be signatures of pulsational pair instability (Rakavy \& Shaviv 1967; Barkat et al. 1967; Woosley et al. 2007). The pulsational pair-instability scenario discussed by Woosley et al. (2007) is applicable for stars with main-sequence masses in the range 95-130 $M_{\odot}$. This is apparently above the mass proposed for the precursor of SN 2009ip. We note, however, that the absolute magnitude of the LBV progenitor of SN 2009ip (see, e.g., Figure 3 in Foley et al. 2011) is also consistent with evolutionary tracks of stellar masses that are much higher than $60 M_{\odot}$, and so should be regarded as a lower mass limit.

The Woosley et al. (2007) model of a pulsational pairinstability SN suggests that colliding shells of material can dissipate most of the relative kinetic energy as radiation. One 
solar mass of material moving at $8000 \mathrm{~km} \mathrm{~s}^{-1}$ has a kinetic energy of more than $10^{50} \mathrm{erg}$, enough to power the measured bolometric light curve of the 2012b event shown in Figure 4. As SN 2009ip has experienced multiple mass ejections, perhaps even more than those we have detected due to possible gaps in the observational coverage (Figure 2), it is plausible there are shells, or clumps of slower moving gas that will slow the fast ejecta of 2009ip during the 2012a episode.

As discussed in Mauerhan et al. (2012a), there are no known line-driven wind mechanism or continuum-driven wind mechanism for driving material off the stellar surface at the high velocities observed. The energy to provide $\gtrsim 10^{50}$ erg per solar mass ejected must presumably come from a core-related event.

There is also some consistency in the velocity of the material ejected during the $2012 \mathrm{a}$ event and the radius of the emitting region in the $2012 \mathrm{~b}$ episode. The $2012 \mathrm{a}$ event lasts approximately 50 days, during which the bulk of material starts at $8000-9000 \mathrm{~km} \mathrm{~s}^{-1}$ on 2012 September 5 , slowing to $5000-6000 \mathrm{~km} \mathrm{~s}^{-1}$ after about 10 days. The fast ejecta likely traveled around $5 \times 10^{4} R_{\odot}$, before impacting on a surrounding shell and causing the dramatic increase in luminosity in the rise to the $2012 \mathrm{~b}$ light curve peak. If the kinetic energy of the shell is then converted into radiative energy, one would expect that an emitting sphere of radius $5 \times 10^{4} R_{\odot}$ at a blackbody temperature of around $10,000 \mathrm{~K}$ would emit at $L \simeq$ a few $\times 10^{43} \mathrm{erg} \mathrm{s}^{-1}$. This crude luminosity estimate is of the same order of magnitude to that we see in Figure 4.

The pulsational pair-instability SN model requires a star of initial mass to be in the range $95-130 M_{\odot}$. The standard massloss prescriptions for such massive stars has to be relaxed so that in the final stages the star should retain enough mass to enhance the core temperature to cause the pair instability. The progenitor has been estimated to have more than $60 M_{\odot}$, implying that it has retained most of its envelope. This is supported by the evidence that broad hydrogen features are detected in all the ejection episodes (Smith et al. 2010; Foley et al. 2011; see also Section 2.3).

An interesting measurement would be the metallicity at the distance of SN 2009ip from the host galaxy nucleus (about $4 \mathrm{kpc}$ ) to determine if the environment is significantly metal poor. At the current stage, only a statistical approach is possible to grossly estimate the local oxygen abundance. Adopting the host galaxy distance and reddening of Smith et al. (2010), the host galaxy has an absolute $B$-band magnitude of -17.9 . Following Pilyugin et al. (2004), the characteristic (at $R=0.4 R_{25}$ ) oxygen abundance of NGC 7259 would be $12+\log (\mathrm{O} / \mathrm{H})=$ 8.34 , which gives $12+\log (\mathrm{O} / \mathrm{H})=8.07$ at the $\mathrm{SN}$ position, clearly sub-solar. Although this method may provide-in the best case-only a rough estimate of the oxygen abundance, a sub-solar metallicity is the natural expectation from the modest host galaxy brightness and the peripheral location of the transient.

As a consequence, a pulsational pair-instability scenario may provide a plausible explanation for the 2012 events, without necessarily invoking the core collapse of the star. According to this, the 2012a event may have been a pulsational pairinstability eruption followed by collisions of these ejecta with pre-existing CSM. The late September-early October spectra of SN 2009ip, again dominated by narrow lines with Lorentzian profiles, indicate that the high-velocity material is covered by electron scattering in a high-density interaction shell (Mauerhan et al. 2012a; Chugai et al. 2004). We note that, as mentioned in Section 1 , there is a relatively weak X-ray emission $\left(L_{X} \sim\right.$
$4 \times 10^{39} \mathrm{erg} \mathrm{s}^{-1}$ at maximum) ${ }^{35}$ and no radio detection of SN 2009ip. Although this would not support strong ejecta-CSM interaction, it does not necessarily rule it out. According to the pulsational pair-instability scenario, the star's core is slowly contracting and is finally expected to become a real corecollapse SN (within a few years) with a potentially very luminous display (Woosley et al. 2007).

\subsection{Was the Historical SN 1961V Similar to the 2012 bruptive Event?}

The photometric comparison between SN 2009ip and SN 1961V shown in Figure 2, including the major eruption when SN 1961V reached an absolute peak magnitude of above -17 , and the spectra (Branch \& Greenstein 1971) dominated by relatively narrow $\mathrm{H}$ lines, suggest a close similarity between these two transients, hence supporting the statement that SN 1961V may have been another pulsational pair-instability event.

SN $1961 \mathrm{~V}$ had a very troublesome genesis. For many years, from 1937 to 1954, its quiescent progenitor was the most luminous star in the host galaxy, NGC 1058. It had an apparent photographic magnitude of 18 (Bertola 1964, corresponding to an absolute magnitude $M_{B} \approx-12$ ). With this luminosity, the star-likely an LBV-had an estimated $M_{\text {ZAMS }}>80 M_{\odot}$ (adopting metallicities from $1 / 3$ to $1 Z_{\odot}$; Kochanek et al. 2011). Then the object was observed at a constant magnitude of about 14.1-14.3 from 1961 July to November, and finally rose to a sharp maximum at mag $\approx 13$ on 1961 December 11 (Bertola 1964, 1967). The peak was followed by a complex luminosity decline, which lasted for a few years with highly variable slopes (see Figure 2).

The nature of this transient has been widely debated, and independent studies gave contradictory results on its real nature (genuine SN or SN impostor; see discussion in Smith et al. 2010). On the one hand, some authors state that a postoutburst surviving star (known as "Object 7"; see Van Dyk \& Matheson 2012 and references therein) is visible in HST optical archival images. The 1961 December light curve peak and the fluctuations in the post-maximum luminosity decline of SN $1961 \mathrm{~V}$ could have been produced by strong interaction between fast-moving, high-density material produced in an eruptive episode before 1961 with a lower-density, pre-existent circumstellar shell, without the need of a proper SN explosion, as also suggested by Van Dyk \& Matheson (2012). On the other hand, on the basis of the lack of sufficient infrared emission from the survived putative progenitor, Kochanek et al. (2011) proposed that SN 1961V had effectively exploded as a real SN, and its unusual observed properties could be explained via the ejecta interacting with a dense circumstellar medium.

Even after half a century from the outburst, we can only speculate about the nature of SN $1961 \mathrm{~V}$, without giving definitive answers. Many years after that event, we tackle an analogous situation. SN 2009ip shares many strong similarities with SN 1961V, and the available information collected for SN 2009ip so far favor the pulsational pair-instability scenario of an extremely massive LBV. Whether the star has ended its life in the final core-collapse SN explosion or the 2012 b re-brightening

\footnotetext{
35 Strongly interacting Type IIn SNe have been observed to reach peak X-ray luminosities $L_{X} \approx 10^{41}-10^{42} \mathrm{erg} \mathrm{s}^{-1}$ (Dwarkadas \& Gruszko 2012 and references therein). As a comparison, stripped-envelope core-collapse $\mathrm{SNe}$ have $L_{X}$ in the range $10^{38}-10^{40} \mathrm{erg} \mathrm{s}^{-1}$, while for SNe IIP, usually $L_{X} \ll 10^{38} \mathrm{erg} \mathrm{s}^{-1}$ is found. We note that the X-ray emission of SN 2009ip is close to that of SN 2011 ht (Roming et al. 2012), whose nature-real SN or SN impostor-has not been firmly established.
} 
event was due to shell-shell collisions is not known yet. Only long-term monitoring of this erupting LBV will perhaps unveil its fate.

\section{CONCLUSIONS}

We presented the results of our spectroscopic and photometric observational campaign for SN 2009ip, spanning a temporal window of more than three years. There is clear evidence from the recent photometric history that, since 2009 August, SN 2009ip was repeatedly seen in outburst. The light curve during the eruptive phases was erratic and reached an absolute peak magnitude of -14 to -15 . More recently, another eruptive episode was observed (the 2012a event), lasting about 50 days and reaching a luminosity of the same order of magnitude of previous outbursts $\left(L \approx 3 \times 10^{41} \mathrm{erg} \mathrm{s}^{-1}\right)$. However, since late 2012 September, another re-brightening was observed (the $2012 \mathrm{~b}$ event), reaching an unprecedented peak luminosity ( $L \approx 8 \times 10^{42} \mathrm{erg} \mathrm{s}^{-1}$ ).

During all these outbursts, the spectra were dominated by strong and relatively narrow $\mathrm{H}$ emission lines, similar to those observed in several SN impostors, but also in Type IIn SNe. In addition, broad P-Cygni absorptions indicative of high-velocity ejecta (up to $14,000-15,000 \mathrm{~km} \mathrm{~s}^{-1}$ ) became prominent during the $2012 \mathrm{a}$ event. We noticed, however, that the signature of highvelocity material was observed in earlier spectra of SN 2009ip, suggesting that the presence of fast ejecta does not necessarily imply a core-collapse SN.

The recent spectrophotometric observations of SN 2009ip and its energetics favor a pulsational pair-instability scenario where collisions among massive shells power the light curve, rather than a genuine SN explosion, although with the available information we cannot definitely rule out that the $2012 \mathrm{a} / \mathrm{b}$ events witnessed the death of the LBV progenitor as a core-collapse SN.

Given this spectacular latest event, it would seem incumbent upon us to secure long-term monitoring campaigns (spectroscopy and imaging from both targeted and archival work) to track the variability history of the SN impostors. These longterm campaigns are probably the most fruitful method to understanding the mechanisms that cause the unpredictable variability and determine the fate of LBVs, the most massive stars in the local universe. SN 2009ip should be one of the best-studied transient events in history. Already, the data collected on the progenitor star outstrip all the information we have on all other SN progenitors to date.

We thank the anonymous referee for a thorough reading of the manuscript and insightful comments. We are grateful to L. Girardi for useful discussions. A.P., E.C., S.B., M.L.P., A.H., L.T., and M.T. are partially supported by the PRIN-INAF 2011 with the project "Transient Universe: from ESO Large to PESSTO." F.B. acknowledges support from FONDECYT through Postdoctoral grant 3120227. M.H., G.P., and F.B. acknowledge support by the Millennium Center for Supernova Science through grant P10-064-F (funded by "Programa Bicentenario de Ciencia y Tecnología de CONICYT" and "Programa Iniciativa Científica Milenio de MIDEPLAN"). This work is supported in part under grant number 1108890 from the US National Science Foundation.

This work is partially based on observations of the European supernova collaboration involved in the ESO-NTT large programme 184.D-1140 led by Stefano Benetti. It is also based on observations made with ESO VLT Telescopes at the Paranal
Observatory under program IDs 087.D-0693 and 089.D-0325 (PI: S. Benetti), 083.D-0131 (PI: S. J. Smartt), and with the ESO-NTT at the La Silla Observatory under program ID 083.D-0970 (PI: S. Benetti).

This paper is based on observations made with the Italian Telescopio Nazionale Galileo (TNG) operated on the island of La Palma by the Fundación Galileo Galilei of the INAF (Istituto Nazionale di Astrofisica). It is also based on observations made with the William Herschel Telescope (WHT) operated on the island of La Palma by the Isaac Newton Group in the Spanish Observatorio del Roque de los Muchachos of the Instituto de Astrofsica de Canarias; the Liverpool Telescope (LT) operated on the island of La Palma at the Spanish Observatorio del Roque de los Muchachos of the Instituto de Astrofisica de Canarias; the Nordic Optical Telescope (NOT), operated on the island of La Palma jointly by Denmark, Finland, Iceland, Norway, and Sweden, in the Spanish Observatorio del Roque de los Muchachos of the Instituto de Astrofísica de Canarias; the $2.2 \mathrm{~m}$ telescope of the Centro Astronómico Hispano Alemán (CAHA) at Calar Alto, operated jointly by the Max-Planck Institut für Astronomie and the Instituto de Astrofísica de Andalucía (CSIC); the $1.82 \mathrm{~m}$ Copernico telescope of INAFAsiago Observatory; the Gemini Observatory, which is operated by the Association of Universities for Research in Astronomy, Inc., under a cooperative agreement with the NSF on behalf of the Gemini partnership; and the Panchromatic Robotic Optical Monitoring and Polarimetry (PROMPT) Telescopes, which are by the National Science Foundation, the University of North Carolina at Chapel Hill, Leonard Goodman, the National Aeronautics and Space Administration, Dudley Observatory, Henry Cox, and the Pisgah Astronomical Research Institute.

The paper also presents observations obtained with the Faulkes Telescope South. The Faulkes Telescopes are maintained and operated by Las Cumbres Observatory Global Telescope Network.

This publication makes use of data products from the Two Micron All Sky Survey, which is a joint project of the University of Massachusetts and the Infrared Processing and Analysis Center/California Institute of Technology, funded by the National Aeronautics and Space Administration and the National Science Foundation.

Facilities: NTT (ESO), VLT:Antu, VLT:Kueyen, TNG, Liverpool:2m, NOT, CTIO:PROMPT, Gemini:Gillett, Swift, FTS

\section{REFERENCES}

Barkat, Z., Rakavy, G., \& Sack, N. 1967, PhRvL, 18, 379

Berger, E., Foley, R., \& Ivans, I. 2009, ATel, 2184, 1

Bertola, F. 1963, CoAsi, 142, 3

Bertola, F. 1964, AnAp, 27, 319

Bertola, F. 1965, CoAsi, 171, 3

Bertola, F. 1967, IBVS, 196, 1

Bohlsen, T. C. 2012, ATel, 4477, 1

Branch, D., \& Greenstein, J. L. 1971, ApJ, 167, 89

Brimacombe, J. 2012, ATel, 4423, 1

Brown, P. J., Dessart, L., Holland, S. T., et al. 2007, ApJ, 659, 1488

Bufano, F., Pian, E., Sollerman, J., et al. 2012, ApJ, 753, 67

Burgasser, A., Nicholls, C., \& Aberasturi, M. 2012, ATel, 4431, 1

Campana, S. 2012, ATel, 4444, 1

Campana, S., \& Margutti, R. 2012, ATel, 4499, 1

Chandra, P., \& Soderberg, A. M. 2012, ATel, 4433, 1

Chu, Y.-H., Gruendl, R. A., Stockdale, C. J., et al. 2004, AJ, 127, 2850

Chugai, N. N., Blinnikov, S. I., Cumming, R. J., et al. 2004, MNRAS, 352, 1213

Davidson, K., \& Humphreys, R. M. 1997, ARA\&A, 35, 1

Dessart, L., Hillier, D. J., Gezari, S., Basa, S., \& Matheson, T. 2009, MNRAS, 394, 21 
Di Carlo, E., Massi, F., Valentini, G., et al. 2002, ApJ, 573, 114 Drake, A. J., Howerton, S., McNaught, R., et al. 2012, ATel, 4334, 1 Drake, A. J., Prieto, J. L., Djorgovski, S. G., et al. 2010, ATel, 2897, 1 Dwarkadas, V. V., \& Gruszko, J. 2012, MNRAS, 419, 1515

Fassia, A., Meikle, W. P. S., Vacca, W. D., et al. 2000, MNRAS, 318, 1093 Foley, R. J., Berger, E., Fox, O., et al. 2011, ApJ, 732, 32

Foley, R. J., Berger, E., Roederer, I. U., \& Chomiuk, L. 2012, ATel, 4338, 1 Foley, R. J., Smith, N., Ganeshalingam, M., et al. 2007, ApJL, 657, L105

Gall, C., Hjorth, J., \& Leloudas, G. 2012, ATel, 4454, 1

Gal-Yam, A., \& Leonard, D. C. 2009, Natur, 458, 865

Gal-Yam, A., Leonard, D. C., Fox, D. B., et al. 2007, ApJ, 656, 372

Gerardy, C. L., Fesen, R. A., Nomoto, K., et al. 2002, ApJ, 575, 1007

Hancock, P., Bannister, K., \& Bell, M. 2012, ATel, 4434, 1

Humphreys, R. M., \& Davidson, K. 1994, PASP, 106, 1025

Humphreys, R. M., Davidson, K., Jones, T. J., et al. 2012, ApJ, 760, 93

Humphreys, R. M., Davidson, K., \& Smith, N. 1999, PASP, 111, 1124

Jha, S. W., McCully, C., \& Patel, B. 2012, ATel, 4491, 1

Kankare, E., Ergon, M., Bufano, F., et al. 2012, MNRAS, 424, 855

Kochanek, C. S., Szczygiel, D. M., \& Stanek, K. Z. 2011, ApJ, 737, 76

Kotak, R., \& Vink, J. S. 2006, A\&A, 460, L5

Landolt, A. U. 1992, AJ, 104, 340

Leonard, D. C., Fedrow, J. M., Khandrika, H. G., \& Gonzalez, L. 2012, ATel, 4430,1

Levesque, E. M., Stringfellow, G. S., Ginsburg, A. G., Bally, J., \& Keeney, B. A. 2012, ApJ, submitted (arXiv:1211.4577)

Li, W., Smith, N., Miller, A. A., \& Filippenko, A. V. 2009, ATel, 2212, 1

Liu, Q.-Z., Hu, J.-Y., Hang, H.-R., et al. 2000, A\&AS, 144, 219

Margutti, R., \& Soderberg, A. 2012, ATel, 4457, 1

Margutti, R., Soderberg, A., Chornock, R., \& Foley, R. 2012a, ATel, 4425, 1

Margutti, R., Soderberg, A., \& Milisavljevic, D. 2012b, ATel, 4414, 1

Martin, J. C., Hambsch, F.-J., \& Tan, T.-G. 2012a, ATel, 4445, 1

Martin, J. C., O'Brien, J., \& Hubbell-Thomas, J. 2012b, ATel, 4416, 1

Mauerhan, J. C., Smith, N., Filippenko, A., et al. 2012a, MNRAS, in press (arXiv:1209.6320)

Mauerhan, J. C., Smith, N., Silverman, J. M., et al. 2012b, MNRAS, submitted (arXiv:1209.0821)

Maund, J. R., Smartt, S. J., Kudritzki, R.-P., et al. 2006, MNRAS, 369, 390

Maza, J., Hamuy, M., Antezana, R., et al. 2009, CBET, 1928, 1

Miller, A. A., Li, W., Nugent, P. E., et al. 2009, ATel, 2183, 1
Pastorello, A., Botticella, M. T., Trundle, C., et al. 2010, MNRAS, 408, 181 Pastorello, A., Mattila, S., Zampieri, L., et al. 2008a, MNRAS, 389, 113 Pastorello, A., Quimby, R. M., Smartt, S. J., et al. 2008b, MNRAS, 389, 131

Pastorello, A., Sauer, D., Taubenberger, S., et al. 2006, MNRAS, 370, 1752

Pastorello, A., Smartt, S. J., Mattila, S., et al. 2007, Natur, 447, 829

Pastorello, A., Valenti, S., Zampieri, L., et al. 2009, MNRAS, 394, 2266

Pastorello, A., Zampieri, L., Turatto, M., et al. 2004, MNRAS, 347, 74

Pilyugin, L. S., Vílchez, J. M., \& Contini, T. 2004, A\&A, 425, 849

Poole, T. S., Breeveld, A. A., Page, M. J., et al. 2008, MNRAS, 383, 627

Pozzo, M., Meikle, W. P. S., Fassia, A., et al. 2004, MNRAS, 352, 457

Prieto, J. L., Brimacombe, J., \& Drake, A. J. 2012, ATel, 4439, 1

Prieto, J. L., Brimacombe, J., Drake, A. J., \& Howerton, S. 2013, ApJL, 763, L27

Rakavy, G., \& Shaviv, G. 1967, ApJ, 148, 803

Roming, P. W. A., Pritchard, T. A., Prieto, J. L., et al. 2012, ApJ, 751, 92

Skrutskie, M. F., Cutri, R. M., Stiening, R., et al. 2006, AJ, 131, 1163

Smith, N. 2008, Natur, 455, 201

Smith, N., \& Frew, D. J. 2011, MNRAS, 415, 2009

Smith, N., Li, W., Foley, R. J., et al. 2007, AJ, 666, 1116

Smith, N., Li, W., Miller, A. A., et al. 2011a, ApJ, 732, 63

Smith, N., Li, W., Silverman, J. M., Ganeshalingam, M., \& Filippenko, A. V. 2011b, MNRAS, 415, 773

Smith, N., \& Mauerhan, J. C. 2012a, ATel, 4412, 1

Smith, N., \& Mauerhan, J. C. 2012b, ATel, 4427, 1

Smith, N., Mauerhan, J. C., Silverman, J. M., et al. 2012, MNRAS, 426, 1905

Smith, N., Miller, A., Li, W., et al. 2010, AJ, 139, 1451

Smith, N., \& Owocki, S. P. 2006, ApJL, 645, L45

Soker, N., \& Kashi, A. 2013, ApJL, 764, L6

Trundle, C., Kotak, R., Vink, J. S., \& Meikle, W. P. S. 2008, A\&A, 483, L47

Trundle, C., Pastorello, A., Benetti, S., et al. 2009, A\&A, 504, 945

Tylenda, R. 2005, A\&A, 436, 1009

Tylenda, R., \& Soker, N. 2006, A\&A, 451, 223

Van Dyk, S. D., \& Matheson, T. 2012, AJ, 143, 19

Van Dyk, S. D., Peng, C. Y., King, J. Y., et al. 2000, PASP, 112, 1532

Vink, J. S., \& de Koter, A. 2002, A\&A, 393, 543

Vinko, J. 2012, ATel, 4480, 1

Vinko, J., Wheeler, J. C., Milisavljevic, D., et al. 2012, ATel, 4435, 1

Wagner, R. M., Vrba, F. J., Henden, A. A., et al. 2004, PASP, 116, 326

Woosley, S. F., Blinnikov, S., \& Heger, A. 2007, Natur, 450, 390 